\title{
ON THE DISCRETENESS OF TRANSMISSION EIGENVALUES FOR THE MAXWELL EQUATIONS*
}

\author{
FIORALBA CAKONI ${ }^{\dagger}$ AND HOAI-MINH NGUYEN ${ }^{\ddagger}$
}

\begin{abstract}
In this paper, we establish the discreteness of transmission eigenvalues for Maxwell's equations. More precisely, we show that the spectrum of the transmission eigenvalue problem is discrete if the electromagnetic parameters $\varepsilon, \mu, \hat{\varepsilon}, \hat{\mu}$ in the equations characterizing the inhomogeneity and background are smooth in some neighborhood of the boundary and isotropic on the boundary, and satisfy the conditions $\varepsilon \neq \hat{\varepsilon}, \mu \neq \hat{\mu}$, and $\varepsilon / \mu \neq \hat{\varepsilon} / \hat{\mu}$ on the boundary. These are quite general assumptions on the coefficients, which are easy to check. To our knowledge, our paper is the first to establish discreteness of transmission eigenvalues for Maxwell's equations without assuming any restrictions on the sign combination of the contrasts $\varepsilon-\hat{\varepsilon}$ and $\mu-\hat{\mu}$ near the boundary and allowing for all the electromagnetic parameters to be inhomogeneous and anisotropic, except for on the boundary where they are isotropic but not necessarily constant as is often assumed in the literature.
\end{abstract}

Key words. transmission eigenvalues, inverse scattering, spectral theory, Cauchy's problem, complementing conditions

AMS subject classifications. 35A01, 35A15, 78A25, 78A46

DOI. $10.1137 / 20 \mathrm{M} 1335121$

1. Introduction. The transmission eigenvalue problem is at the heart of inverse scattering theory for inhomogeneous media. This eigenvalue problem is a late arrival in scattering theory with its first appearance in $[11,18]$, in connection with the injectivity of the relative scattering operator. Transmission eigenvalues are related to interrogating frequencies for which there is an incident field that doesn't scatterer by the medium. The transmission eigenvalue problem has a deceptively simple formulation, namely, two elliptic PDEs in a bounded domain (one governs the wave propagation in the scattering medium and the other in the background that occupies the support of the medium) that share the same Cauchy data on the boundary, but presents a perplexing mathematical structure. In particular, it is a non-self-adjoint eigenvalue problem for a nonstrongly elliptic operator, and hence the investigation of its spectral properties becomes challenging. Roughly, the spectral properties depend on the assumptions on the contrasts in the media (i.e., the difference of the respective coefficients in each of the equations) near the boundary. Questions central to the inverse scattering theory include discreteness of the spectrum that is closely related to the determination of the support of inhomogeneity from scattering data using linear sampling and factorization methods [5], location of transmission eigenvalues in the complex plane that is essential to the development of the time domain linear sampling method [9], and the existence of transmission eigenvalues as well as the accurate determination of real transmission eigenvalues from scattering data, which has become important since real transmission eigenvalues could be used to obtain information

\footnotetext{
${ }^{*}$ Received by the editors April 30, 2020; accepted for publication October 19, 2020; published electronically February 4, 2021.

https://doi.org/10.1137/20M1335121

Funding: The work of the first author was partially supported by the AFOSR grant FA955020-1-0024 and NSF grant DMS-1813492.

$\dagger^{\dagger}$ Department of Mathematics, Rutgers University, New Brunswick, NJ 08903 USA (fc292@math. rutgers.edu).

${ }^{\ddagger}$ Ecole Polytechnique Fédérale de Lausanne, EPFL, SB, CAMA, Station 8, CH-1015 Lausanne, Switzerland (hoai-minh.nguyen@epfl.ch).
} 
about the material properties of the scattering media. We refer the reader to [5] for a recent and self-contained introduction to the topic.

This paper concerns the discreteness and location of transmission eigenvalues in the scattering of time-harmonic electromagnetic waves by an inhomogeneous (possibly anisotropic) medium of bounded support. Let us introduce the mathematical formulation of the electromagnetic transmission eigenvalue problem. To this end, let $\Omega$ be an open, bounded subset of $\mathbb{R}^{3}$ representing the support of the inhomogeneity, which we assume to be of class $C^{2}$. Let $\varepsilon, \mu, \hat{\varepsilon}, \hat{\mu}$ be $(3 \times 3)$ symmetric, uniformly elliptic, matrix-valued functions defined in $\Omega$ with $L^{\infty}(\Omega)$ entries. A complex number $\omega$ is called an eigenvalue of the transmission eigenvalue problem, or a transmission eigenvalue, associated with $\varepsilon, \mu, \hat{\varepsilon}, \hat{\mu}$ in $\Omega$ if there exists a nonzero solution $(E, H, \hat{E}, \hat{H}) \in\left[L^{2}(\Omega)\right]^{12}$ of the following system:

$$
\begin{aligned}
& \left\{\begin{array}{c}
\nabla \times E=i \omega \mu H, \\
\nabla \times H=-i \omega \varepsilon E
\end{array} \text { in } \Omega, \quad\left\{\begin{array}{c}
\nabla \times \hat{E}=i \omega \hat{\mu} \hat{H}, \\
\nabla \times \hat{H}=-i \omega \hat{\varepsilon} \hat{E}
\end{array} \quad \text { in } \Omega,\right.\right. \\
& (\hat{E}-E) \times \nu=0 \text { on } \partial \Omega, \quad \text { and } \quad(\hat{H}-H) \times \nu=0 \text { on } \partial \Omega,
\end{aligned}
$$

where $\nu$ denotes the outward unit normal vector to $\partial \Omega$.

The main result that we prove in this paper is stated in Theorem 1.1 below. For the reader's convenience we first must clarify some terminology used in the formulation of this theorem. A $3 \times 3$ matrix-valued function $M$ defined in a subset $O \subset \mathbb{R}^{3}$ is called isotropic at $x \in O$ if it is proportional to the identity matrix at $x$, i.e., $M(x)=m I$ for some scalar $m=m(x)$, where $I$ denotes the $3 \times 3$ identity matrix. In this case, for notational ease, we also denote $m(x)$ by $M(x)$. If $M$ is isotropic for $x \in O$, then $M$ is said to be isotropic in $O$. Condition (1.3) below is understood under the convention $m(x)=M(x)$.

Theorem 1.1. Assume that

(i) $\varepsilon, \mu, \hat{\varepsilon}, \hat{\mu}$ are of class $C^{1}$ in some neighborhood of $\partial \Omega$,

(ii) $\varepsilon, \mu, \hat{\varepsilon}, \hat{\mu}$ are isotropic on $\partial \Omega$,

$$
\varepsilon \neq \hat{\varepsilon}, \quad \mu \neq \hat{\mu}, \quad \varepsilon / \mu \neq \hat{\varepsilon} / \hat{\mu} \quad \text { on } \partial \Omega .
$$

The set of the transmission eigenvalues of (1.1) and (1.2) is discrete with $\infty$ as the only possible accumulation point.

The analysis used in the proof of Theorem 1.1 also allows us to obtain the following result on the transmission eigenvalue free region of the complex plane $\mathbb{C}$.

Proposition 1.1. Assume that $\varepsilon, \mu, \hat{\varepsilon}, \hat{\mu}$ are of class $C^{1}$ in some neighborhood of $\partial \Omega$, isotropic on $\partial \Omega$, and

$$
\varepsilon \neq \hat{\varepsilon}, \quad \mu \neq \hat{\mu}, \quad \varepsilon / \mu \neq \hat{\varepsilon} / \hat{\mu} \quad \text { on } \partial \Omega
$$

For $\gamma>0$, there exists $\omega_{0}>0$ such that if $\omega \in \mathbb{C}$ with $\left|\Im\left(\omega^{2}\right)\right| \geq \gamma|\omega|^{2}$ and $|\omega| \geq \omega_{0}$, then $\omega$ is not a transmission eigenvalue.

Here and and in what follows, for $z \in \mathbb{C}$, let $\Im(z)$ denote the imaginary part of $z$.

Remark 1.1. Since $\gamma>0$ can be chosen arbitrarily small, the result of Proposition 1.1, together with the fact that $\infty$ is the only accumulation point of the transmission eigenvalues proven in Theorem 1.1, implies that all the transmission eigenvalues $\omega$, but finitely many, lie in two wedges of an arbitrarily small angle. 
The structure of the spectrum of the transmission eigenvalue problem is better understood in the case of scalar inhomogeneous Helmoltz equations. In this case, the transmission eigenvalue problem can be stated as follows. Let $d \geq 2$ and $\Omega$ be an open, bounded Lipschitz subset of $\mathbb{R}^{d}$. Let $A_{1}, A_{2}$ be two $(d \times d)$ symmetric, uniformly elliptic, matrix-valued functions defined in $\Omega$ and $\Sigma_{1}$ and $\Sigma_{2}$ be two bounded positive functions defined in $\Omega$. A complex number $\omega$ is called an eigenvalue of the transmission eigenvalue problem, or a transmission eigenvalue, if there exists a nonzero solution $\left(u_{1}, u_{2}\right)$ of the system

$$
\begin{gathered}
\begin{cases}\operatorname{div}\left(A_{1} \nabla u_{1}\right)+\omega^{2} \Sigma_{1} u_{1}=0 & \text { in } \Omega, \\
\operatorname{div}\left(A_{2} \nabla u_{2}\right)+\omega^{2} \Sigma_{2} u_{2}=0 & \text { in } \Omega,\end{cases} \\
u_{1}=u_{2}, \quad A_{1} \nabla u_{1} \cdot \nu=A_{2} \nabla u_{2} \cdot \nu
\end{gathered}
$$

The discreteness of transmission eigenvalues for the Helmholtz equation has been investigated extensively in the literature. The first discreteness result appeared in [29], whereas [26] proves the state-of-the-art results on the discreteness of transmission eigenvalues for anisotropic background and inhomogeneity under most general assumptions on the coefficients using Fourier and multiplier approaches. More specifically, it is shown in [26] that the transmission eigenvalue problem has a discrete spectrum if the coefficients are smooth only near the boundary and

(i) $A_{1}(x), A_{2}(x)$ satisfy the complementing boundary condition with respect to $\nu(x)$ for all $x \in \partial \Omega$, i.e., for all $x \in \partial \Omega$ and for all $\xi \in \mathbb{R}^{d} \backslash\{0\}$ with $\xi \cdot \nu=0$, we have

$$
\left\langle A_{2} \nu, \nu\right\rangle\left\langle A_{2} \xi, \xi\right\rangle-\left\langle A_{2} \nu, \xi\right\rangle^{2} \neq\left\langle A_{1} \nu, \nu\right\rangle\left\langle A_{1} \xi, \xi\right\rangle-\left\langle A_{1} \nu, \xi\right\rangle^{2},
$$

(ii) $\left\langle A_{1} \nu, \nu\right\rangle \Sigma_{1} \neq\left\langle A_{2} \nu, \nu\right\rangle \Sigma_{2}$ for all $x \in \partial \Omega$.

Additional results in [26] also include various combinations of the sign of contrasts $A_{1}-A_{2}$ and $\Sigma_{1}-\Sigma_{2}$ on the boundary. Previous results on discreteness can be found in $[3,19,32]$ and references therein. We must emphasize that the conditions (i) and (ii) are more general than simply one sign contrasts $A_{2}-A_{1}$ and/or $\Sigma_{2}-\Sigma_{1}$ near the boundary. To complete the picture on the transmission eigenvalue problem in the scalar case, we remark that the first answer to the existence of transmission eigenvalues for one sign contrast in $\Omega$ was given in [28], where the authors showed the existence of a few real transmission eigenvalues for the index of refraction sufficiently large, followed by $[6,8]$, which prove the existence of infinite real transmission eigenvalues removing the size restriction on the index. The completeness of transmission eigenfunctions and first estimates on the counting function are shown in $[30,31]$ for $C^{\infty}$ boundary and coefficients since they use semiclassical analysis and pseudodifferential calculus. Again in a $C^{\infty}$ setting, $[33,34]$ prove the sharpest known results in the scalar case on eigenvalue free zones and Weyl's law for the scalar case improving an earlier result by $[17]$.

The story of the transmission eigenvalue problem for Maxwell's equations is not as complete as for the scalar case discussed above. One of the first results on discreteness is given by Haddar in [14], which considers the case of $\mu=\hat{\varepsilon}=\hat{\mu}=I$, and $\varepsilon-I$ invertible in $\Omega$. Chesnel in [10] employs the so-called $T$-coercivity to prove discreteness when $\hat{\varepsilon}=\hat{\mu}=I$, and $\varepsilon-I$ and $\mu^{-1}-I$ are both greater than $c I$ or both less than $-c I$ for some positive constant $c$ in a neighborhood of $\partial \Omega$. Cakoni, Haddar, and Meng in [7] use an integral equation approach to study discreteness for the case when 
$\mu=\hat{\varepsilon}=\hat{\mu}=I$ and the matrix-valued $\varepsilon$ becomes a constant not equal to 1 near the boundary. Theorem 1.1 therefore adds to this list quite general conditions on the coefficients for which the discreteness holds. To our knowledge, our paper is the first to establish discreteness of transmission eigenvalues for Maxwell's equation under sign assumptions on the contrast $\epsilon-\hat{\epsilon}$ and $\mu-\hat{\mu}$ on the boundary, and allowing for all the electromagnetic parameters to be inhomogeneous and anisotropic, except for on the boundary where they are isotropic but not necessarily constant as is often assumed in the literature. For the case of electromagnetic transmission eigenvalue problems, other types of results are rather limited, and we refer the reader to [6] for the existence of real transmission eigenvalues and [16] for the completeness of eigenfunctions for the setting related to the one in [7] mentioned above.

The analysis in this paper is inspired by the concept of complementary conditions suggested by Agmon, Douglis, and Nirenberg in their celebrated papers [1,2] for elliptic systems. For Maxwell's equations, the complementary condition for the Cauchy problems has been recently investigated in [27] for general anisotropic coefficients in the context of negative index metamaterials. To be able to apply the theory of complementing conditions to the Maxwell equations, various forms of the Poincaré lemma and Helmholtz decomposition are used with a suitable implementation of local charts. The analysis in this paper is in the spirit of the one developed in [26]. The idea is to show that the system

$$
\begin{gathered}
\left\{\begin{array} { c } 
{ \nabla \times E = i \omega \mu H + J _ { e } \text { in } \Omega , } \\
{ \nabla \times H = - i \omega \varepsilon E + J _ { m } \text { in } \Omega , }
\end{array} \quad \left\{\begin{array}{c}
\nabla \times \hat{E}=i \omega \hat{\mu} \hat{H}+\hat{J}_{e} \text { in } \Omega, \\
\nabla \times \hat{H}=-i \omega \hat{\varepsilon} \hat{E}+\hat{J}_{m} \text { in } \Omega,
\end{array}\right.\right. \\
(\hat{E}-E) \times \nu=0 \text { on } \partial \Omega, \quad \text { and } \quad(\hat{H}-H) \times \nu=0 \text { on } \partial \Omega,
\end{gathered}
$$

is well-posed for some $\omega \in \mathbb{C}$, where $\left(J_{e}, J_{m}, \hat{J}_{e}, \hat{J}_{m}\right)$ is the input, which belongs to an appropriate functional space. Moreover, a key fact is to prove that the corresponding transformation which maps the input $\left(J_{e}, J_{m}, \hat{J}_{e}, \hat{J}_{m}\right)$ to the output $(E, H, \hat{E}, \hat{H})$ is compact. It is worth mentioning that the compactness is one of the crucial/critical differences between the study of the Maxwell equations and the Helmholtz equation. In our analysis, the functional space for the input is well-chosen so that the compactness property holds (see (4.22)) for $\omega$ in some domain. For example, these facts hold under the assumptions of Theorem 1.1 provided that $i \omega=|\omega| e^{i \pi / 4}$, i.e., $\omega=|\omega| e^{-i \pi / 4}$ and $|\omega|$ is large. To this end, we analyze the corresponding Cauchy problem with constant coefficients in a half-space (Proposition 3.1). Using the decay of Maxwell equations (Lemma 4.1), we can prove the uniqueness for (1.7) and (1.8). To establish the existence of a solution, the Banach-Necas-Babuska theorem is applied. Deriving (1.3) and handling the compactness are the key differences in the analysis of this paper and the one for the scalar case [26].

The Cauchy problem also naturally appears in the context of negative index materials after using reflections as initiated in [21]. The well-posedness and the limiting absorption principle for the Helmholtz equations with sign-changing coefficients were developed in [22] using the Fourier and multiplier approach. Recently, with Sil, the second author investigated these problems for the Maxwell equations [27]. Both papers $[22,27]$ deal with the stability question of negative index materials and are the starting point for the analysis of the discreteness of transmission eigenvalues for the Helmholtz equation [26] and Maxwell's equations in this work. Other aspects and applications of negative index materials involving the stability and instability of the Cauchy problem (1.7) and (1.8) are discussed in [23, 25, 24] and the references therein. 
The paper is organized as follows. In section 2, we introduce notation used frequently in this paper. Section 3 is devoted to the analysis in the half space. The main result in this section is Proposition 3.1. Condition (1.3) will appear very naturally there. Finally, we present the proof of Theorem 1.1 in section 4 . The choice of the right functional space plays an important role there. The proof of Proposition 1.1 is also given in this section.

2. Notation. The following notation is used frequently throughout the paper. Denote

$$
\mathbb{R}_{+}^{3}=\left\{x=\left(x_{1}, x_{2}, x_{3}\right) \in \mathbb{R}^{3} ; x_{3}>0\right\}
$$

and

$$
\mathbb{R}_{0}^{3}=\left\{x=\left(x_{1}, x_{2}, x_{3}\right) \in \mathbb{R}^{3} ; x_{3}=0\right\} .
$$

Let $\Omega$ be a bounded, open subset of $\mathbb{R}^{3}$ and of class $C^{2}$, or $\Omega=\mathbb{R}_{+}^{3}$. We define the spaces

$$
\begin{gathered}
H(\operatorname{curl}, \Omega)=\left\{u \in\left[L^{2}(\Omega)\right]^{3} ; \nabla \times u \in\left[L^{2}(\Omega)\right]^{3}\right\}, \\
H_{0}(\operatorname{curl}, \Omega)=\{u \in H(\operatorname{curl}, \Omega) ; u \times \nu=0 \text { on } \partial \Omega\}, \\
H(\operatorname{div}, \Omega)=\left\{u \in\left[L^{2}(\Omega)\right]^{3} ; \operatorname{div} u \in L^{2}(\Omega)\right\} .
\end{gathered}
$$

Set $\Gamma=\partial \Omega$, and for $s=-1 / 2$, or $1 / 2$, define the trace space

$$
H_{\text {div }}^{s}(\Gamma)=\left\{u \in\left[H^{s}(\Gamma)\right]^{3} ; u \cdot \nu=0 \text { and } \operatorname{div}_{\Gamma} u \in H^{s}(\Gamma)\right\} .
$$

For a vector field $u$ defined in a subset of $\mathbb{R}^{3}, u_{j}$ denotes its $j$ th component for $1 \leq j \leq 3$. We also denote, for $s>0$,

$$
\Omega_{s}=\{x \in \Omega ; \operatorname{dist}(x, \partial \Omega)<s\} .
$$

3. Analysis on a half space. In order to simplify presentation, we let $k \in \mathbb{C}$ be $k:=i \omega$. Let $\varepsilon, \mu, \hat{\varepsilon}, \hat{\mu}$ be four symmetric, uniformly elliptic matrix-valued functions defined in $\mathbb{R}_{+}^{3}$. In this section, we are interested in the following Cauchy problem for Maxwell's equations in $\mathbb{R}_{+}^{3}$, with $J_{e}, J_{m}, \hat{J}_{e}, \hat{J}_{m} \in L^{2}\left(\mathbb{R}_{+}^{3}\right)$ and $f_{e}, f_{m} \in H_{\mathrm{div}}^{-1 / 2}\left(\mathbb{R}_{0}^{3}\right)$,

$$
\left\{\begin{array} { c } 
{ \nabla \times E = k \mu H + J _ { e } \text { in } \mathbb { R } _ { + } ^ { 3 } , } \\
{ \nabla \times H = - k \varepsilon E + J _ { m } \text { in } \mathbb { R } _ { + } ^ { 3 } , }
\end{array} \quad \left\{\begin{array}{c}
\nabla \times \hat{E}=k \hat{\mu} \hat{H}+\hat{J}_{e} \text { in } \mathbb{R}_{+}^{3}, \\
\nabla \times \hat{H}=-k \hat{\varepsilon} \hat{E}+\hat{J}_{m} \text { in } \mathbb{R}_{+}^{3},
\end{array}\right.\right.
$$

and $^{1}$

$$
(\hat{E}-E) \times e_{3}=f_{e} \text { on } \mathbb{R}_{0}^{3}, \quad \text { and } \quad(\hat{H}-H) \times e_{3}=f_{m} \text { on } \mathbb{R}_{0}^{3} .
$$

We begin with proving the following lemma.

\footnotetext{
${ }^{1} e_{3}=(0,0,1) \in \mathbb{R}^{3}$.
} 
Lemma 3.1. Let $\gamma>0$ and $k \in \mathbb{C}$ with $\left|\Im\left(k^{2}\right)\right| \geq \gamma|k|^{2}$ and $|k| \geq 1$. Furthermore, let $\Lambda \geq 1$ and $\varepsilon, \mu$ be two positive constants such that $\Lambda^{-1} \leq \varepsilon, \mu \leq \Lambda$. For $J_{e}, J_{m} \in$ $\left[L^{2}\left(\mathbb{R}_{+}^{3}\right)\right]^{3}$, there exists a unique solution $(E, H) \in\left[L^{2}\left(\mathbb{R}^{3}\right)\right]^{6}$ of the system

$$
\left\{\begin{array}{c}
\nabla \times E=k \mu H \text { in } \mathbb{R}_{+}^{3}, \\
\nabla \times H=-k \varepsilon E+J_{m} \text { in } \mathbb{R}_{+}^{3}, \\
E \times e_{3}=0 \text { on } \mathbb{R}_{0}^{3} .
\end{array}\right.
$$

Moreover, for some positive constant $C$ depending only on $\Lambda$ and $\gamma$,

$$
\|(E, H)\|_{L^{2}\left(\mathbb{R}_{+}^{3}\right)} \leq \frac{C}{|k|}\left\|J_{m}\right\|_{L^{2}\left(\mathbb{R}_{+}^{3}\right)}
$$

and

$$
\left(\int_{\mathbb{R}^{2}}\left|H^{\mathcal{F}}(\xi) \times e_{3}\right|^{2}\left(|k|^{2}+|\xi|^{2}\right)^{-1 / 2} d \xi\right)^{1 / 2} \leq \frac{C}{|k|}\left\|J_{m}\right\|_{L^{2}\left(\mathbb{R}_{+}^{3}\right)}
$$

and if $\operatorname{div} J_{m} \in L^{2}(\Omega)$, then

$$
\|(E, H)\|_{H^{1}\left(\mathbb{R}_{+}^{3}\right)} \leq C\left(\left\|J_{m}\right\|_{L^{2}\left(\mathbb{R}_{+}^{3}\right)}+\frac{1}{|k|}\left\|\operatorname{div} J_{m}\right\|_{L^{2}\left(\mathbb{R}_{+}^{3}\right)}\right) .
$$

Here and in what follows, we denote the Fourier transform with respect to $\left(x_{1}, x_{2}\right) \in \mathbb{R}^{2}$ of an appropriate function $u: \mathbb{R}_{+}^{3} \rightarrow \mathbb{C}$ by $u^{\mathcal{F}}$, i.e.,

$$
u^{\mathcal{F}}\left(\xi, x_{3}\right)=\frac{1}{2 \pi} \int_{\mathbb{R}^{2}} u\left(x_{1}, x_{2}, x_{3}\right) e^{-i\left(x_{1} \xi_{1}+x_{2} \xi_{2}\right)} d x_{1} d x_{2} \text { for }\left(\xi, x_{3}\right)=\left(\xi_{1}, \xi_{2}, x_{3}\right) \in \mathbb{R}_{+}^{3} .
$$

Similar notation is used for an appropriate function defined on $\mathbb{R}_{0}^{3}$. We also identity a vector $\left(y_{1}, y_{2}, 0\right) \in \mathbb{R}_{0}^{3}$ with $\left(y_{1}, y_{2}\right) \in \mathbb{R}^{2}$.

Remark 3.1. We emphasize here that the constant $C$ appearing in (3.4)-(3.6) is independent of $k$.

Proof. We have, from the system of $(E, H)$,

$$
\nabla \times(\nabla \times E)+k^{2} \varepsilon \mu E=k \mu J_{m} \text { in } \mathbb{R}_{+}^{3} .
$$

Multiplying (3.7) by $\bar{\varphi}$ (the conjugate of $\varphi$ ) with $\varphi \in H_{0}\left(\right.$ curl, $\left.\mathbb{R}_{+}^{3}\right)$ and integrating by parts yields

$$
\int_{\mathbb{R}_{+}^{3}}\langle\nabla \times E, \nabla \times \varphi\rangle+k^{2} \varepsilon \mu \int_{\mathbb{R}^{3}}\langle E, \varphi\rangle=\int_{\mathbb{R}^{3}} k \mu\left\langle J_{m}, \varphi\right\rangle
$$

Take $\varphi=E$. Since $\left|\Im\left(k^{2}\right)\right| \geq \gamma|k|^{2}$ and $|k| \geq 1$, after considering the imaginary part and the real part of (3.8), we obtain

$$
\int_{\mathbb{R}_{+}^{3}}|\nabla \times E|^{2}+|k|^{2}|E|^{2} \leq C \int_{\mathbb{R}_{+}^{3}}\left|J_{m}\right|^{2}
$$

which implies (3.4) since $\nabla \times E=k \mu H$ in $\mathbb{R}_{+}^{3}$. The uniqueness of $(E, H)$ follows.

Copyright $@$ by SIAM. Unauthorized reproduction of this article is prohibited. 
To derive (3.6), we note that

$$
\begin{gathered}
\|\nabla \times E\|_{L^{2}\left(\mathbb{R}_{+}^{3}\right)} \leq C\left\|J_{m}\right\|_{L^{2}\left(\mathbb{R}_{+}^{3}\right)}, \\
\|\operatorname{div} E\|_{L^{2}\left(\mathbb{R}_{+}^{3}\right)} \leq \frac{C}{|k|}\left\|\operatorname{div} J_{m}\right\|_{L^{2}\left(\mathbb{R}_{+}^{3}\right)}, \\
\|E\|_{L^{2}\left(\mathbb{R}_{+}^{3}\right)} \leq C\left\|J_{m}\right\|_{L^{2}\left(\mathbb{R}_{+}^{3}\right)}, \\
E \times e_{3}=0 \text { on } \mathbb{R}_{+}^{3} .
\end{gathered}
$$

It follows from the Gaffney inequality (see, e.g., [13, Theorem 3.7], [12, Theorem 1]) that $\left.E \in H^{1}\left(\mathbb{R}_{+}^{3}\right)\right]^{3}$ and

$$
\|E\|_{H^{1}\left(\mathbb{R}_{+}^{3}\right)} \leq C\left\|J_{m}\right\|_{L^{2}\left(\mathbb{R}_{+}^{3}\right)}+\frac{C}{|k|}\left\|\operatorname{div} J_{m}\right\|_{L^{2}\left(\mathbb{R}_{+}^{3}\right)} .
$$

We also have

$$
\begin{gathered}
\|\nabla \times H\|_{L^{2}\left(\mathbb{R}_{+}^{3}\right)} \leq C\left\|J_{m}\right\|_{L^{2}\left(\mathbb{R}_{+}^{3}\right)}, \\
\|\operatorname{div} H\|_{L^{2}\left(\mathbb{R}_{+}^{3}\right)}=0 \\
\|H\|_{L^{2}\left(\mathbb{R}_{+}^{3}\right)} \leq C\left\|J_{m}\right\|_{L^{2}\left(\mathbb{R}_{+}^{3}\right)},
\end{gathered}
$$

and, since $E \times e_{3}=0$ on $\mathbb{R}_{+}^{3}$,

$$
H \cdot e_{3}=0 \text { on } \mathbb{R}_{+}^{3} .
$$

It follows from the Gaffney inequality again (see, e.g., [13, Theorem 3.9], [12, Theorem 1]) that $H \in H^{1}\left(\mathbb{R}_{+}^{3}\right)$ and

$$
\|H\|_{H^{1}\left(\mathbb{R}_{+}^{3}\right)} \leq C\left\|J_{m}\right\|_{L^{2}\left(\mathbb{R}_{+}^{3}\right)}+\frac{C}{|k|}\left\|\operatorname{div} J_{e}\right\|_{L^{2}\left(\mathbb{R}_{+}^{3}\right)} .
$$

Combining (3.9) and (3.10) yields (3.6).

To establish (3.5), we proceed as follows. Set, in $\mathbb{R}_{+}^{3}$,

$$
(\widetilde{E}, \widetilde{H})(x)=(E, H)(x /|k|) \quad \text { and } \quad \widetilde{J}_{m}(x)=\frac{1}{|k|} J_{m}(x /|k|) .
$$

We then have

$$
\left\{\begin{array}{c}
\nabla \times \widetilde{E}=\frac{k}{|k|} \mu \widetilde{H} \text { in } \mathbb{R}_{+}^{3} \\
\nabla \times \widetilde{H}=-\frac{k}{|k|} \varepsilon \widetilde{E}+\widetilde{J}_{m} \text { in } \mathbb{R}_{+}^{3} \\
\widetilde{E} \times e_{3}=0 \text { on } \mathbb{R}_{0}^{3} .
\end{array}\right.
$$

Applying (3.4) to $(\widetilde{E}, \widetilde{H})$, we have

$$
\|(\widetilde{E}, \widetilde{H})\|_{L^{2}\left(\mathbb{R}_{+}^{3}\right)} \leq C\left\|\widetilde{J}_{m}\right\|_{L^{2}\left(\mathbb{R}_{+}^{3}\right)}=C|k|^{1 / 2}\left\|J_{m}\right\|_{L^{2}\left(\mathbb{R}_{+}^{3}\right)} \quad \text { (by a change of variables). }
$$

This implies, by the trace theory,

$$
\left\|\widetilde{H} \times e_{3}\right\|_{H^{-1 / 2}\left(\mathbb{R}_{0}^{3}\right)} \leq C\|\widetilde{H}\|_{H\left(\operatorname{curl}, \mathbb{R}_{+}^{3}\right)} \leq C\left\|\widetilde{J}_{m}\right\|_{L^{2}\left(\mathbb{R}_{+}^{3}\right)}=C|k|^{1 / 2}\left\|J_{m}\right\|_{L^{2}\left(\mathbb{R}_{+}^{3}\right)} .
$$

Copyright $@$ ( ) by SIAM. Unauthorized reproduction of this article is prohibited. 
Note that

$$
\widetilde{H}^{\mathcal{F}}(\xi)=|k|^{2} H^{\mathcal{F}}(|k| \xi) \text { for } \xi \in \mathbb{R}^{2} .
$$

It follows that

$$
\begin{aligned}
\left\|\widetilde{H} \times e_{3}\right\|_{H^{-1 / 2}\left(\mathbb{R}_{0}^{3}\right)}^{2} & =\int_{\mathbb{R}^{2}}\left|\widetilde{H}^{\mathcal{F}}(\xi) \times e_{3}\right|^{2}\left(1+|\xi|^{2}\right)^{-1 / 2} d \xi \\
& =\int_{\mathbb{R}^{2}}|k|^{4}\left|H^{\mathcal{F}}(|k| \xi) \times e_{3}\right|^{2}\left(1+|\xi|^{2}\right)^{-1 / 2} d \xi
\end{aligned}
$$

This yields, by a change of variables,

$$
\left\|\widetilde{H} \times e_{3}\right\|_{H^{-1 / 2}\left(\mathbb{R}_{0}^{3}\right)}^{2}=\int_{\mathbb{R}^{2}}|k|^{3}\left|H^{\mathcal{F}}(\xi) \times e_{3}\right|^{2}\left(|k|^{2}+|\xi|^{2}\right)^{-1 / 2} d \xi .
$$

From (3.12) and (3.13), we obtain

$$
\int_{\mathbb{R}^{2}}\left|H^{\mathcal{F}}(\xi) \times e_{3}\right|^{2}\left(|k|^{2}+|\xi|^{2}\right)^{-1 / 2} d \xi \leq C|k|^{-2}\left\|J_{m}\right\|_{L^{2}\left(\mathbb{R}_{+}^{3}\right)}^{2}
$$

Assertion (3.5) follows.

To prove the existence of $(E, H)$, we first apply the Lax-Milgram theory for the variational formula given in (3.8), where the bilinear form is defined by the left hand side (LHS) and the linear functional is defined by the RHS in the Hilbert space $H_{0}\left(\operatorname{curl}, \mathbb{R}_{+}^{3}\right)$. We then derive that there exists a solution $E \in H_{0}\left(\operatorname{curl}, \mathbb{R}_{+}^{3}\right)$ of $(3.7)$. Set

$$
H=\frac{1}{k \mu} \nabla \times E \text { in } \mathbb{R}_{+}^{3} .
$$

Then

$$
\nabla \times E=k \mu H \text { in } \mathbb{R}_{+}^{3}
$$

and

$$
\nabla \times H=\frac{1}{k \mu} \nabla \times(\nabla \times E)=-k \varepsilon E+J_{m} \text { in } \mathbb{R}_{+}^{3} .
$$

In other words, $(E, H) \in\left[L^{2}\left(\mathbb{R}_{+}^{3}\right)\right]^{6}$ is a solution of (3.3). The proof is complete.

We now state the main result of this section, which plays a key role in the proof of Theorem 1.1.

Proposition 3.1. Let $\gamma>0, k \in \mathbb{C}$ with $\left|\Im\left(k^{2}\right)\right| \geq \gamma|k|^{2}$, and $|k| \geq 1$, and let $\Lambda \geq 1$ and $\varepsilon, \mu, \hat{\varepsilon}, \hat{\mu}$ be four positive constants such that

$$
\Lambda^{-1} \leq \varepsilon, \mu, \hat{\varepsilon}, \hat{\mu} \leq \Lambda .
$$

Assume that, for some $\Lambda_{1}>0$,

$$
|\varepsilon-\hat{\varepsilon}| \geq \Lambda_{1}, \quad|\mu-\hat{\mu}| \geq \Lambda_{1}, \quad \text { and } \quad|\varepsilon / \mu-\hat{\varepsilon} / \hat{\mu}| \geq \Lambda_{1} .
$$

Let $J_{e}, J_{m}, \hat{J}_{e}, \hat{J}_{m} \in L^{2}\left(\mathbb{R}_{+}^{3}\right)$ and let $f_{e}, f_{m} \in H^{-1 / 2}\left(\operatorname{div}, \mathbb{R}_{0}^{3}\right)$. There exists a unique solution $(E, H, \hat{E}, \hat{H}) \in\left[L^{2}\left(\mathbb{R}_{+}^{3}\right)\right]^{12}$ of the system

$$
\left\{\begin{array} { c } 
{ \nabla \times E = k \mu H + J _ { e } \text { in } \mathbb { R } _ { + } ^ { 3 } , } \\
{ \nabla \times H = - k \varepsilon E + J _ { m } \text { in } \mathbb { R } _ { + } ^ { 3 } , }
\end{array} \quad \left\{\begin{array}{c}
\nabla \times \hat{E}=k \hat{\mu} \hat{H}+\hat{J}_{e} \text { in } \mathbb{R}_{+}^{3}, \\
\nabla \times \hat{H}=-k \hat{\varepsilon} \hat{E}+\hat{J}_{m} \text { in } \mathbb{R}_{+}^{3},
\end{array}\right.\right.
$$

Copyright $@$ by SIAM. Unauthorized reproduction of this article is prohibited. 


$$
(\hat{E}-E) \times e_{3}=f_{e} \text { on } \mathbb{R}_{0}^{3}, \quad \text { and } \quad(\hat{H}-H) \times e_{3}=f_{m} \text { on } \mathbb{R}_{0}^{3} .
$$

Moreover, we have

$$
\begin{aligned}
& C|k|\|(E, H, \hat{E}, \hat{H})\|_{L^{2}\left(\mathbb{R}_{+}^{3}\right)} \leq\left\|\left(J_{e}, J_{m}, \hat{J}_{e}, \hat{J}_{m}\right)\right\|_{L^{2}\left(\mathbb{R}_{+}^{3}\right)} \\
+ & |k|\left(\int_{\mathbb{R}^{2}}\left|\left(f_{e}^{\mathcal{F}}, f_{m}^{\mathcal{F}}\right)\right|^{2}\left(|\xi|^{2}+|k|^{2}\right)^{-1 / 2} d \xi\right)^{1 / 2}+\left\|\left(\operatorname{div}_{\Gamma} f_{e}, \operatorname{div}_{\Gamma} f_{m}\right)\right\|_{H^{-1 / 2}\left(\mathbb{R}_{0}^{3}\right)} .
\end{aligned}
$$

Assume in addition that $J_{e}, J_{m}, \hat{J}_{e}, \hat{J}_{m} \in H\left(\operatorname{div}, \mathbb{R}_{+}^{3}\right)$ with $\left(J_{e, 3}-\hat{J}_{e, 3}, J_{m, 3}-\hat{J}_{m, 3}\right) \in$ $\left[H^{1 / 2}\left(\mathbb{R}_{0}^{3}\right)\right]^{2}$, and $f_{e}, f_{m} \in H^{1 / 2}\left(\operatorname{div}, \mathbb{R}_{0}^{3}\right)$. We have

$$
\begin{aligned}
C\left(\|(E, H, \hat{E}, \hat{H})\|_{H^{1}\left(\mathbb{R}_{+}^{3}\right)}+|k|\|(E, H, \hat{E}, \hat{H})\|_{L^{2}\left(\mathbb{R}_{+}^{3}\right)}\right) \\
\leq \quad\left\|\left(J_{e}, J_{m}, \hat{J}_{e}, \hat{J}_{m}\right)\right\|_{L^{2}\left(\mathbb{R}_{+}^{3}\right)}+\frac{1}{|k|}\left\|\left(\operatorname{div} J_{e}, \operatorname{div} J_{m}, \operatorname{div} \hat{J}_{e}, \operatorname{div} \hat{J}_{m}\right)\right\|_{L^{2}\left(\mathbb{R}_{+}^{3}\right)} \\
\quad+\frac{1}{|k|}\left\|\left(J_{e, 3}-\hat{J}_{e, 3}, J_{m, 3}-\hat{J}_{m, 3}\right)\right\|_{H^{1 / 2}\left(\mathbb{R}_{0}^{3}\right)}+|k|^{1 / 2}\left\|\left(f_{e}, f_{m}\right)\right\|_{L^{2}\left(\mathbb{R}_{0}^{3}\right)} \\
+\left\|\left(f_{e}, f_{m}\right)\right\|_{H^{1 / 2}\left(\mathbb{R}_{0}^{3}\right)}+\frac{1}{|k|}\left\|\left(\operatorname{div}_{\Gamma} f_{e}, \operatorname{div}_{\Gamma} f_{m}\right)\right\|_{H^{1 / 2}\left(\mathbb{R}_{0}^{3}\right)} .
\end{aligned}
$$

Here $C$ denotes a positive constant depending only on $\gamma, \Lambda$, and $\Lambda_{1}$.

Recall that, by our convention, $J_{e, 3}, J_{m, 3}, \hat{J}_{e, 3}, \hat{J}_{m, 3}$ denote the third component of $J_{e}, J_{m}, \hat{J}_{e}, \hat{J}_{m}$. It is worth noting that the constant $C$ is independent of $k$.

Proof. Let $\left(E^{1}, H^{1}\right),\left(E^{2}, H^{2}\right),\left(\hat{E}^{1}, \hat{H}^{1}\right),\left(\hat{E}^{2}, \hat{H}^{2}\right) \in\left[L^{2}\left(\mathbb{R}_{+}^{3}\right)\right]^{6}$ be, respectively, the unique solutions of the following systems:

$$
\begin{gathered}
\left\{\begin{array} { c } 
{ \nabla \times E ^ { 1 } = k \mu H ^ { 1 } \text { in } \mathbb { R } _ { + } ^ { 3 } , } \\
{ \nabla \times H ^ { 1 } = - k \varepsilon E ^ { 1 } + J _ { m } \text { in } \mathbb { R } _ { + } ^ { 3 } , } \\
{ E ^ { 1 } \times e _ { 3 } = 0 \text { on } \mathbb { R } _ { 0 } ^ { 3 } , }
\end{array} \left\{\begin{array}{c}
\nabla \times \hat{E}^{1}=k \hat{\mu} \hat{H}^{1} \text { in } \mathbb{R}_{+}^{3}, \\
\nabla \times \hat{H}^{1}=-k \hat{\varepsilon} \hat{E}^{1}+\hat{J}_{m} \text { in } \mathbb{R}_{+}^{3}, \\
\hat{E}^{1} \times e_{3}=0 \text { on } \mathbb{R}_{0}^{3},
\end{array}\right.\right. \\
\left\{\begin{array} { c } 
{ \nabla \times E ^ { 2 } = k \mu H ^ { 2 } + J _ { e } \text { in } \mathbb { R } _ { + } ^ { 3 } , } \\
{ \nabla \times H ^ { 2 } = - k \varepsilon E ^ { 1 } \text { in } \mathbb { R } _ { + } ^ { 3 } , } \\
{ H ^ { 2 } \times e _ { 3 } = 0 \text { on } \mathbb { R } _ { 0 } ^ { 3 } , }
\end{array} \quad \left\{\begin{array}{c}
\nabla \times \hat{E}^{2}=k \hat{\mu} \hat{H}^{2}+\hat{J}_{e} \text { in } \mathbb{R}_{+}^{3}, \\
\nabla \times \hat{H}^{2}=-k \hat{\varepsilon} \hat{E}^{2} \text { in } \mathbb{R}_{+}^{3}, \\
\hat{H}^{2} \times e_{3}=0 \text { on } \mathbb{R}_{0}^{3} .
\end{array}\right.\right.
\end{gathered}
$$

We first establish (3.18). Applying Lemma 3.1, we obtain

$$
\begin{aligned}
& \left\|\left(E^{1}, H^{1}, E^{2}, H^{2}, \hat{E}^{1}, \hat{H}^{1}, \hat{E}^{2}, \hat{H}^{2}\right)\right\|_{H^{1}\left(\mathbb{R}_{+}^{3}\right)} \\
& \quad+|k|\left\|\left(E^{1}, H^{1}, E^{2}, H^{2}, \hat{E}^{1}, \hat{H}^{1}, \hat{E}^{2}, \hat{H}^{2}\right)\right\|_{L^{2}\left(\mathbb{R}_{+}^{3}\right)} \\
& \leq C\left(\left\|\left(J_{e}, J_{m}, \hat{J}_{e}, \hat{J}_{m}\right)\right\|_{L^{2}\left(\mathbb{R}_{+}^{3}\right)}+\frac{1}{|k|}\left\|\left(\operatorname{div} J_{e}, \operatorname{div} J_{m}, \operatorname{div} \hat{J}_{e}, \operatorname{div} \hat{J}_{m}\right)\right\|_{L^{2}\left(\mathbb{R}_{+}^{3}\right)}\right) .
\end{aligned}
$$

Copyright $@$ by SIAM. Unauthorized reproduction of this article is prohibited. 
Using the trace theory, we derive from (3.19) that

$$
\begin{aligned}
& \left\|\left(E^{1}, H^{1}, E^{2}, H^{2}, \hat{E}^{1}, \hat{H}^{1}, \hat{E}^{2}, \hat{H}^{2}\right)\right\|_{H^{1 / 2}\left(\mathbb{R}_{0}^{3}\right)} \\
& \leq C\left\|\left(E^{1}, H^{1}, E^{2}, H^{2}, \hat{E}^{1}, \hat{H}^{1}, \hat{E}^{2}, \hat{H}^{2}\right)\right\|_{H^{1}\left(\mathbb{R}_{+}^{3}\right)} \\
& \leq C\left(\left\|\left(J_{e}, J_{m}, \hat{J}_{e}, \hat{J}_{m}\right)\right\|_{L^{2}\left(\mathbb{R}_{+}^{3}\right)}+\frac{1}{|k|}\left\|\left(\operatorname{div} J_{e}, \operatorname{div} J_{m}, \operatorname{div} \hat{J}_{e}, \operatorname{div} \hat{J}_{m}\right)\right\|_{L^{2}\left(\mathbb{R}_{+}^{3}\right)}\right) .
\end{aligned}
$$

We have

$$
\begin{aligned}
\| \operatorname{div}_{\Gamma}\left(H^{1} \times e_{3}\right) & -\operatorname{div}_{\Gamma}\left(\hat{H}^{1} \times e_{3}\right) \|_{H^{1 / 2}\left(\mathbb{R}_{0}^{3}\right)} \\
& =\left\|\left(\nabla \times H^{1}\right) \cdot e_{3}-\left(\nabla \times \hat{H}^{1}\right) \cdot e_{3}\right\|_{H^{1 / 2}\left(\mathbb{R}_{0}^{3}\right)} \\
& =\left\|\left(-k \varepsilon E^{1}+J_{m}\right) \cdot e_{3}-\left(-k \hat{\varepsilon} \hat{E}^{1}+\hat{J}_{m}\right) \cdot e_{3}\right\|_{H^{1 / 2}\left(\mathbb{R}_{0}^{3}\right)} \\
& \leq C|k|\left\|\left(E^{1}, \hat{E}^{1}\right)\right\|_{H^{1 / 2}\left(\mathbb{R}_{0}^{3}\right)}+C\left\|J_{m, 3}-\hat{J}_{m, 3}\right\|_{H^{1 / 2}\left(\mathbb{R}_{0}^{3}\right)} .
\end{aligned}
$$

It follows from (3.20) that

$$
\begin{gathered}
\left\|\operatorname{div}_{\Gamma}\left(H^{1} \times e_{3}\right)-\operatorname{div}_{\Gamma}\left(\hat{H}^{1} \times e_{3}\right)\right\|_{H^{1 / 2}\left(\mathbb{R}_{0}^{3}\right)} \leq C|k|\left(\left\|\left(J_{e}, J_{m}, \hat{J}_{e}, \hat{J}_{m}\right)\right\|_{L^{2}\left(\mathbb{R}_{+}^{3}\right)}\right. \\
\left.+\frac{1}{|k|}\left\|\left(\operatorname{div} J_{e}, \operatorname{div} J_{m}, \operatorname{div} \hat{J}_{e}, \operatorname{div} \hat{J}_{m}\right)\right\|_{L^{2}\left(\mathbb{R}_{+}^{3}\right)}+\frac{1}{|k|}\left\|J_{m, 3}-\hat{J}_{m, 3}\right\|_{H^{1 / 2}\left(\mathbb{R}_{0}^{3}\right)}\right) .
\end{gathered}
$$

Similarly, we obtain

$$
\begin{array}{r}
\left\|\operatorname{div}_{\Gamma}\left(E^{1} \times e_{3}\right)-\operatorname{div}_{\Gamma}\left(\hat{E}^{1} \times e_{3}\right)\right\|_{H^{1 / 2}\left(\mathbb{R}_{0}^{3}\right)} \leq C|k|\left(\left\|\left(J_{e}, J_{m}, \hat{J}_{e}, \hat{J}_{m}\right)\right\|_{L^{2}\left(\mathbb{R}_{+}^{3}\right)}\right. \\
\left.+\frac{1}{|k|}\left\|\left(\operatorname{div} J_{e}, \operatorname{div} J_{m}, \operatorname{div} \hat{J}_{e}, \operatorname{div} \hat{J}_{m}\right)\right\|_{L^{2}\left(\mathbb{R}_{+}^{3}\right)}+\frac{1}{|k|}\left\|J_{e, 3}-\hat{J}_{e, 3}\right\|_{H^{1 / 2}\left(\mathbb{R}_{0}^{3}\right)}\right) .
\end{array}
$$

Using the fact, for $u \in H^{1}\left(\mathbb{R}_{+}^{3}\right)$,

$$
\int_{\mathbb{R}_{0}^{3}}|u|^{2} \leq 2 \int_{\mathbb{R}_{+}^{3}}|u|\left|\partial_{x_{3}} u\right| \leq 2\|u\|_{L^{2}\left(\mathbb{R}_{+}^{3}\right)}\|\nabla u\|_{L^{2}\left(\mathbb{R}_{+}^{3}\right)},
$$

we have

$$
\begin{aligned}
& \left\|\left(E^{1}, H^{1}, E^{2}, H^{2}, \hat{E}^{1}, \hat{H}^{1}, \hat{E}^{2}, \hat{H}^{2}\right)\right\|_{L^{2}\left(\mathbb{R}_{0}^{3}\right)} \\
& \leq C\left\|\left(E^{1}, H^{1}, E^{2}, H^{2}, \hat{E}^{1}, \hat{H}^{1}, \hat{E}^{2}, \hat{H}^{2}\right)\right\|_{L^{2}\left(\mathbb{R}_{+}^{3}\right)}^{1 / 2} \\
& \times\left\|\left(E^{1}, H^{1}, E^{2}, H^{2}, \hat{E}^{1}, \hat{H}^{1}, \hat{E}^{2}, \hat{H}^{2}\right)\right\|_{H^{1}\left(\mathbb{R}_{+}^{3}\right)}^{1 / 2} .
\end{aligned}
$$

This yields

$$
\begin{aligned}
& k^{1 / 2}\left\|\left(E^{1}, H^{1}, E^{2}, H^{2}, \hat{E}^{1}, \hat{H}^{1}, \hat{E}^{2}, \hat{H}^{2}\right)\right\|_{L^{2}\left(\mathbb{R}_{0}^{3}\right)} \\
& \quad \leq C|k|\left\|\left(E^{1}, H^{1}, \hat{E}^{1}, \hat{H}^{1}\right)\right\|_{L^{2}\left(\mathbb{R}_{+}^{3}\right)}+C\left\|\left(E^{1}, H^{1}, \hat{E}^{1}, \hat{H}^{1}\right)\right\|_{H^{1}\left(\mathbb{R}_{+}^{3}\right)} .
\end{aligned}
$$

Copyright $@$ ( ) by SIAM. Unauthorized reproduction of this article is prohibited. 
By considering $\left(E-E^{1}-E^{2}, H-H^{1}-H^{2}, \hat{E}-\hat{E}^{1}-\hat{E}^{2}, \hat{H}-\hat{H}^{1}-\hat{H}^{2}\right)$, from (3.19), (3.20), (3.21), (3.22), and (3.24), without loss of generality, one might assume that

$$
J_{e}=J_{m}=\hat{J}_{e}=\hat{J}_{m}=0 \text { in } \mathbb{R}_{+}^{3} .
$$

This will be assumed later on. Thus

$$
\begin{aligned}
& \left\{\begin{array} { c } 
{ \nabla \times E = k \mu H \text { in } \mathbb { R } _ { + } ^ { 3 } , } \\
{ \nabla \times H = - k \varepsilon E \text { in } \mathbb { R } _ { + } ^ { 3 } , }
\end{array} \quad \left\{\begin{array}{c}
\nabla \times \hat{E}=k \hat{\mu} \hat{H} \text { in } \mathbb{R}_{+}^{3}, \\
\nabla \times \hat{H}=-k \hat{\varepsilon} \hat{E} \text { in } \mathbb{R}_{+}^{3},
\end{array}\right.\right. \\
& (\hat{E}-E) \times e_{3}=f_{e} \text { on } \mathbb{R}_{0}^{3}, \quad \text { and } \quad(\hat{H}-H) \times e_{3}=f_{m} \text { on } \mathbb{R}_{0}^{3} .
\end{aligned}
$$

Using the identity for a vector field $A$

$$
\nabla \times(\nabla \times A)=\nabla(\nabla \cdot A)-\Delta A,
$$

we obtain the following equations for $E$ and $\hat{E}$ :

$$
\left\{\begin{array}{c}
\Delta E-k^{2} \varepsilon \mu E=0 \text { in } \mathbb{R}_{+}^{3}, \\
\Delta \hat{E}-k^{2} \hat{\varepsilon} \hat{\mu} \hat{E}=0 \text { in } \mathbb{R}_{+}^{3}
\end{array}\right.
$$

(recall that here the coefficients are all constants).

Consider the first two equations of the system for $E$ and the first two equations of the system for $\hat{E}$ in (3.27). Solving these equations using the Fourier transform with respect to $\left(x_{1}, x_{2}\right)$ yields

$$
\begin{array}{ll}
E_{j}^{\mathcal{F}}\left(\xi, x_{3}\right)=a_{j}(\xi) e^{-x_{3} \sqrt{|\xi|^{2}+k^{2} \varepsilon \mu}} & \text { in } \mathbb{R}_{+}^{3}, \\
\hat{E}_{j}^{\mathcal{F}}\left(\xi, x_{3}\right)=\hat{a}_{j}(\xi) e^{-x_{3} \sqrt{|\xi|^{2}+k^{2} \hat{\varepsilon} \hat{\mu}}} \quad \text { in } \mathbb{R}_{+}^{3}
\end{array}
$$

for $j=1,2$, where

$$
a_{j}(\xi)=E_{j}^{\mathcal{F}}(\xi, 0) \quad \text { and } \quad \hat{a}_{j}(\xi)=\hat{E}_{j}^{\mathcal{F}}(\xi, 0) \text { for } \xi \in \mathbb{R}^{2} .
$$

We then have, with $a=\left(a_{1}, a_{2}\right)$ and $\hat{a}=\left(\hat{a}_{1}, \hat{a}_{2}\right)$,

$$
\hat{a}(\xi)-a(\xi)=h(\xi), \text { where } h(\xi)=-f_{e}^{\mathcal{F}}(\xi, 0) \times e_{3} .
$$

Recall that we identify a vector $\left(y_{1}, y_{2}, 0\right) \in \mathbb{R}_{0}^{3}$ with $\left(y_{1}, y_{2}\right) \in \mathbb{R}^{2}$.

Since $\operatorname{div} E=0$ in $\mathbb{R}_{+}^{3}$, it follows that

$$
\partial_{x_{3}} E_{3}=-\left(\partial_{x_{1}} E_{1}+\partial_{x_{2}} E_{2}\right) \quad \text { in } \mathbb{R}_{+}^{3} .
$$

This implies

$$
\partial_{x_{3}} E_{3}^{\mathcal{F}}\left(\xi, x_{3}\right)=-i \xi_{1} E_{1}^{\mathcal{F}}\left(\xi, x_{3}\right)-i \xi_{2} E_{2}^{\mathcal{F}}\left(\xi, x_{3}\right) \quad \text { in } \mathbb{R}_{+}^{3} .
$$

Using (3.28), we obtain

$$
\partial_{x_{3}} E_{3}^{\mathcal{F}}\left(\xi, x_{3}\right)=-i \xi \cdot a(\xi) e^{-x_{3} \sqrt{|\xi|^{2}+k^{2} \varepsilon \mu}} \quad \text { in } \mathbb{R}_{+}^{3} .
$$

Copyright $@$ by SIAM. Unauthorized reproduction of this article is prohibited. 
We thus get

$$
E_{3}^{\mathcal{F}}\left(\xi, x_{3}\right)=-\int_{x_{3}}^{\infty} i \xi \cdot a(\xi) e^{-s \sqrt{|\xi|^{2}+k^{2} \varepsilon \mu}} d s=\frac{i \xi \cdot a(\xi) e^{-x_{3} \sqrt{|\xi|^{2}+k^{2} \varepsilon \mu}}}{\sqrt{|\xi|^{2}+k^{2} \varepsilon \mu}} \quad \text { in } \mathbb{R}_{+}^{3} .
$$

Similarly, we have

$$
\hat{E}_{3}^{\mathcal{F}}\left(\xi, x_{3}\right)=\frac{i \xi \cdot \hat{a}(\xi) e^{-x_{3} \sqrt{|\xi|^{2}+k^{2} \hat{\varepsilon} \hat{\mu}}}}{\sqrt{|\xi|^{2}+k^{2} \hat{\varepsilon} \hat{\mu}}} \quad \text { in } \mathbb{R}_{+}^{3} .
$$

Since $\hat{H} \times e_{3}-H \times e_{3}=f_{m}$ on $\mathbb{R}_{0}^{3}$, and $\nabla \times H=-k \varepsilon E$ and $\nabla \times \hat{H}=-k \hat{\varepsilon} \hat{E}$ in $\mathbb{R}_{+}^{3}$, it follows that

$$
\hat{\varepsilon} \hat{E}_{3}-\varepsilon E_{3}=-\frac{1}{k} \operatorname{div}_{\mathbb{R}_{0}^{3}} f_{m} \quad \text { on } \mathbb{R}_{0}^{3} .
$$

Using (3.31) and (3.32), we derive that

$$
\frac{\hat{\varepsilon} \xi \cdot \hat{a}(\xi)}{\sqrt{|\xi|^{2}+k^{2} \hat{\varepsilon} \hat{\mu}}}-\frac{\varepsilon \xi \cdot a(\xi)}{\sqrt{|\xi|^{2}+k^{2} \varepsilon \mu}}=g:=\left(\frac{i}{k} \operatorname{div}_{\mathbb{R}_{0}^{3}} f_{m}\right)^{\mathcal{F}} \quad \text { on } \mathbb{R}^{2} .
$$

Combining (3.30) and (3.33), and noting $a=\hat{a}-h$, yields, on $\mathbb{R}^{2}$,

$$
\xi \cdot \hat{a}\left(\frac{\hat{\varepsilon}}{\sqrt{|\xi|^{2}+k^{2} \hat{\varepsilon} \hat{\mu}}}-\frac{\varepsilon}{\sqrt{|\xi|^{2}+k^{2} \varepsilon \mu}}\right)=-\frac{\varepsilon}{\sqrt{|\xi|^{2}+k^{2} \varepsilon \mu}} \xi \cdot h+g,
$$

which implies

$$
\begin{aligned}
\xi \cdot \hat{a}=\frac{\sqrt{|\xi|^{2}+k^{2} \varepsilon \mu} \sqrt{|\xi|^{2}+k^{2} \hat{\varepsilon} \hat{\mu}}\left(\varepsilon \sqrt{|\xi|^{2}+k^{2} \hat{\varepsilon} \hat{\mu}}+\hat{\varepsilon} \sqrt{|\xi|^{2}+k^{2} \varepsilon \mu}\right)}{\left(\hat{\varepsilon}^{2}-\varepsilon^{2}\right)|\xi|^{2}+k^{2} \varepsilon \hat{\varepsilon} \mu \hat{\mu}(\hat{\varepsilon} / \hat{\mu}-\varepsilon / \mu)} \\
\times\left(-\frac{\varepsilon}{\sqrt{|\xi|^{2}+k^{2} \varepsilon \mu}} \xi \cdot h+g\right) .
\end{aligned}
$$

Since $\varepsilon \neq \hat{\varepsilon}, \varepsilon / \mu \neq \hat{\varepsilon} / \hat{\mu}$, and $\left|\Im\left(k^{2}\right)\right| \geq \gamma|k|^{2},|k| \geq 1$, we get

$$
\left.\left|\left(\hat{\varepsilon}^{2}-\varepsilon^{2}\right)\right| \xi\right|^{2}+k^{2} \varepsilon \hat{\varepsilon} \mu \hat{\mu}(\hat{\varepsilon} / \hat{\mu}-\varepsilon / \mu) \mid \geq C\left(|\xi|^{2}+|k|^{2}\right) .
$$

We deduce that

$$
|\xi \cdot \hat{a}(\xi)| \leq C\left(|\xi \cdot h(\xi)|+\sqrt{|\xi|^{2}+|k|^{2}}|g(\xi)|\right),
$$

which yields, since $a=\hat{a}-h$,

$$
|\xi \cdot a(\xi)|+|\xi \cdot \hat{a}(\xi)| \leq C\left(|\xi \cdot h(\xi)|+\sqrt{|\xi|^{2}+|k|^{2}}|g(\xi)|\right) .
$$

We have, in $\mathbb{R}_{+}^{3}$,

$$
k \mu H_{1}=\partial_{x_{2}} E_{3}-\partial_{x_{3}} E_{2}, \quad k \hat{\mu} \hat{H}_{1}=\partial_{x_{2}} \hat{E}_{3}-\partial_{x_{3}} \hat{E}_{2} .
$$

Copyright (c) by SIAM. Unauthorized reproduction of this article is prohibited. 
Since $\hat{H}_{1}-H_{1}=f_{m, 2}:=f_{m} \cdot e_{2}$ with $e_{2}=(0,1,0)$ on $\mathbb{R}_{0}^{3}$, it follows from (3.28), (3.29), (3.31), (3.32) that

$$
\begin{aligned}
\frac{1}{\hat{\mu}}\left(-\frac{\xi_{2} \xi \cdot \hat{a}(\xi)}{\sqrt{|\xi|^{2}+k^{2} \hat{\varepsilon} \hat{\mu}}}+\right. & \left.\sqrt{|\xi|^{2}+k^{2} \hat{\varepsilon} \hat{\mu}} \hat{a}_{2}(\xi)\right) \\
& =\frac{1}{\mu}\left(-\frac{\xi_{2} \xi \cdot a(\xi)}{\sqrt{|\xi|^{2}+k^{2} \varepsilon \mu}}+\sqrt{|\xi|^{2}+k^{2} \varepsilon \mu} a_{2}(\xi)\right)+k f_{m, 2}^{\mathcal{F}}(\xi) .
\end{aligned}
$$

We derive from (3.30) that

$$
\begin{aligned}
& \frac{1}{\hat{\mu}} \sqrt{|\xi|^{2}+k^{2} \hat{\varepsilon} \hat{\mu}} \hat{a}_{2}(\xi)-\frac{1}{\mu} \sqrt{|\xi|^{2}+k^{2} \varepsilon \mu} \hat{a}_{2}(\xi) \\
= & \left(\frac{\xi \cdot \hat{a}(\xi)}{\hat{\mu} \sqrt{|\xi|^{2}+k^{2} \hat{\varepsilon} \hat{\mu}}}-\frac{\xi \cdot a(\xi)}{\mu \sqrt{|\xi|^{2}+k^{2} \varepsilon \mu}}\right) \xi_{2}-\frac{1}{\mu} \sqrt{|\xi|^{2}+k^{2} \varepsilon \mu} h_{2}(\xi)+k f_{m, 2}^{\mathcal{F}}(\xi) .
\end{aligned}
$$

We thus obtain

$$
\begin{aligned}
& \hat{a}_{2}(\xi)=\frac{\mu \sqrt{|\xi|^{2}+k^{2} \hat{\varepsilon} \hat{\mu}}+\hat{\mu} \sqrt{|\xi|^{2}+k^{2} \varepsilon \mu}}{\left(\mu^{2}-\hat{\mu}^{2}\right)|\xi|^{2}+k^{2} \varepsilon \hat{\varepsilon} \mu \hat{\mu}(\mu / \varepsilon-\hat{\mu} / \hat{\varepsilon})} \\
& \times\left\{\left(\frac{\mu \xi \cdot \hat{a}(\xi)}{\sqrt{|\xi|^{2}+k^{2} \hat{\varepsilon} \hat{\mu}}}-\frac{\hat{\mu} \xi \cdot a(\xi)}{\sqrt{|\xi|^{2}+k^{2} \varepsilon \mu}}\right) \xi_{2}-\hat{\mu} \sqrt{|\xi|^{2}+k^{2} \varepsilon \mu} h_{2}(\xi)+k \mu \hat{\mu} f_{m, 2}^{\mathcal{F}}(\xi)\right\} .
\end{aligned}
$$

Since $\mu \neq \hat{\mu}, \varepsilon / \mu \neq \hat{\varepsilon} / \hat{\mu}$, and $\left|\Im\left(k^{2}\right)\right| \geq \gamma|k|^{2},|k| \geq 1$, we get

$$
\left.\left|\left(\mu^{2}-\hat{\mu}^{2}\right)\right| \xi\right|^{2}+k^{2} \varepsilon \hat{\varepsilon} \mu \hat{\mu}(\mu / \varepsilon-\hat{\mu} / \hat{\varepsilon}) \mid \geq C\left(|\xi|^{2}+|k|^{2}\right) .
$$

Using (3.38), we derive from (3.37) that

$$
\left|\hat{a}_{2}(\xi)\right| \leq \frac{C|\xi|}{|\xi|^{2}+|k|^{2}}(|\xi \cdot a(\xi)|+|\xi \cdot \hat{a}(\xi)|)+C\left(\left|h_{2}(\xi)\right|+\left|f_{m, 2}^{\mathcal{F}}(\xi)\right|\right),
$$

which yields, since $\hat{a}-a=h$,

$$
\left|a_{2}(\xi)\right|+\left|\hat{a}_{2}(\xi)\right| \leq \frac{C|\xi|}{|\xi|^{2}+|k|^{2}}(|\xi \cdot a(\xi)|+|\xi \cdot \hat{a}(\xi)|)+C\left(|h(\xi)|+\left|f_{m}^{\mathcal{F}}(\xi)\right|\right) .
$$

Combining (3.35) and (3.39) yields

$$
\left(\left|a_{2}(\xi)\right|^{2}+\left|\hat{a}_{2}(\xi)\right|^{2}\right) \sqrt{|\xi|^{2}+|k|^{2}} \leq C\left(|h|^{2}+\left|f_{m}^{\mathcal{F}}\right|^{2}\right) \sqrt{|\xi|^{2}+|k|^{2}}+C|g(\xi)|^{2}|\xi| .
$$

From the definition of $g$ and $h$, we obtain

$$
\begin{aligned}
\int_{\mathbb{R}^{2}}\left(\left|a_{2}(\xi)\right|^{2}+\left|\hat{a}_{2}(\xi)\right|^{2}\right) \sqrt{|\xi|^{2}+|k|^{2}} d \xi \leq C\left(\left\|\left(f_{e}, f_{m}\right)\right\|_{H^{1 / 2}\left(\mathbb{R}_{0}^{3}\right)}^{2}\right. \\
\left.+|k|\left\|\left(f_{e}, f_{m}\right)\right\|_{L^{2}\left(\mathbb{R}_{0}^{3}\right)}^{2}+\frac{1}{|k|^{2}}\left\|\left(\operatorname{div}_{\Gamma} f_{e}, \operatorname{div}_{\Gamma} f_{m}\right)\right\|_{H^{1 / 2}\left(\mathbb{R}_{0}^{3}\right)}^{2}\right) .
\end{aligned}
$$

Copyright (c) by SIAM. Unauthorized reproduction of this article is prohibited. 
Similarly, we reach

$$
\begin{aligned}
\int_{\mathbb{R}^{2}}\left(\left|a_{1}(\xi)\right|^{2}+\left|\hat{a}_{1}(\xi)\right|^{2}\right) \sqrt{|\xi|^{2}+|k|^{2}} d \xi \leq C\left(\left\|\left(f_{e}, f_{m}\right)\right\|_{H^{1 / 2}\left(\mathbb{R}_{0}^{3}\right)}^{2}\right. \\
\left.+|k|\left\|\left(f_{e}, f_{m}\right)\right\|_{L^{2}\left(\mathbb{R}_{0}^{3}\right)}^{2}+\frac{1}{|k|^{2}}\left\|\left(\operatorname{div}_{\Gamma} f_{e}, \operatorname{div}_{\Gamma} f_{m}\right)\right\|_{H^{1 / 2}\left(\mathbb{R}_{0}^{3}\right)}^{2}\right) .
\end{aligned}
$$

On the other hand, from (3.28), (3.29), (3.31), and (3.32), we have

$$
\int_{\mathbb{R}_{+}^{3}}|(\nabla E, \nabla \hat{E})|^{2}+|k|^{2}|(E, \hat{E})|^{2} d x \leq C \int_{\mathbb{R}^{2}}|(a(\xi), \hat{a}(\xi))|^{2} \sqrt{|\xi|^{2}+|k|^{2}} d \xi .
$$

Combining (3.40), (3.41), and (3.42) yields

$$
\begin{aligned}
\int_{\mathbb{R}_{+}^{3}}|(\nabla E, \nabla \hat{E})|^{2} & +|k|^{2}|(E, \hat{E})|^{2} d x \leq C\left(\left\|\left(f_{e}, f_{m}\right)\right\|_{H^{1 / 2}\left(\mathbb{R}_{0}^{3}\right)}^{2}\right. \\
& \left.+|k|\left\|\left(f_{e}, f_{m}\right)\right\|_{L^{2}\left(\mathbb{R}_{0}^{3}\right)}^{2}+\frac{1}{|k|^{2}}\left\|\left(\operatorname{div}_{\Gamma} f_{e}, \operatorname{div}_{\Gamma} f_{m}\right)\right\|_{H^{1 / 2}\left(\mathbb{R}_{0}^{3}\right)}^{2}\right) .
\end{aligned}
$$

Similarly, we obtain

$$
\begin{aligned}
\int_{\mathbb{R}_{+}^{3}}|(\nabla H, \nabla \hat{H})|^{2}+|k|^{2}|(H, \hat{H})|^{2} d x \leq C\left(\left\|\left(f_{e}, f_{m}\right)\right\|_{H^{1 / 2}\left(\mathbb{R}_{0}^{3}\right)}^{2}\right. \\
\left.+|k|\left\|\left(f_{e}, f_{m}\right)\right\|_{L^{2}\left(\mathbb{R}_{0}^{3}\right)}^{2}+\frac{1}{|k|^{2}}\left\|\left(\operatorname{div}_{\Gamma} f_{e}, \operatorname{div}_{\Gamma} f_{m}\right)\right\|_{H^{1 / 2}\left(\mathbb{R}_{0}^{3}\right)}^{2}\right) .
\end{aligned}
$$

Assertion (3.18) now follows from (3.43) and (3.44).

We next deal with (3.17), whose proof is in the same spirit. Applying Lemma 3.1, we obtain

$$
|k|\left\|\left(E^{1}, H^{1}, E^{2}, H^{2}, \hat{E}^{1}, \hat{H}^{1}, \hat{E}^{2}, \hat{H}^{2}\right)\right\|_{L^{2}\left(\mathbb{R}_{+}^{3}\right)} \leq C\left\|\left(J_{e}, J_{m}, \hat{J}_{e}, \hat{J}_{m}\right)\right\|_{L^{2}\left(\mathbb{R}_{+}^{3}\right)},
$$

and, with $u=E^{1}, H^{1}, E^{2}, H^{2}, \hat{E}^{1}, \hat{H}^{1}, \hat{E}^{2}$, or $\hat{H}^{2}$,

$$
|k|\left(\int_{\mathbb{R}^{2}}\left|u^{\mathcal{F}}(\xi) \times e_{3}\right|^{2}\left(|\xi|^{2}+|k|^{2}\right)^{-1 / 2} d \xi\right)^{1 / 2} \leq C\left\|\left(J_{e}, J_{m}, \hat{J}_{e}, \hat{J}_{m}\right)\right\|_{L^{2}\left(\mathbb{R}_{+}^{3}\right)} .
$$

As in the proof of (3.21) we obtain

$$
\begin{aligned}
\| \operatorname{div}_{\Gamma}\left(H^{1} \times e_{3}\right) & -\operatorname{div}_{\Gamma}\left(\hat{H}^{1} \times e_{3}\right) \|_{H^{-1 / 2}\left(\mathbb{R}_{0}^{3}\right)} \\
& =\left\|\left(\nabla \times H^{1}\right) \cdot e_{3}-\left(\nabla \times \hat{H}^{1}\right) \cdot e_{3}\right\|_{H^{-1 / 2}\left(\mathbb{R}_{0}^{3}\right)} \\
& =\left\|\left(-k \varepsilon E^{1}+J_{m}\right) \cdot e_{3}-\left(-k \hat{\varepsilon} \hat{E}^{1}+\hat{J}_{m}\right) \cdot e_{3}\right\|_{H^{-1 / 2}\left(\mathbb{R}_{0}^{3}\right)} \\
& \leq C\left\|\left(-k \varepsilon E^{1}+J_{m}\right)-\left(-k \hat{\varepsilon} \hat{E}^{1}+\hat{J}_{m}\right)\right\|_{L^{2}\left(\mathbb{R}_{+}^{3}\right)} \text { (by the trace theory), }
\end{aligned}
$$

Copyright (c) by SIAM. Unauthorized reproduction of this article is prohibited. 
which yields, by (3.45),

$$
\left\|\operatorname{div}_{\Gamma}\left(H^{1} \times e_{3}\right)-\operatorname{div}_{\Gamma}\left(\hat{H}^{1} \times e_{3}\right)\right\|_{H^{-1 / 2}\left(\mathbb{R}_{0}^{3}\right)} \leq C\left\|\left(J_{e}, J_{m}, \hat{J}_{e}, \hat{J}_{m}\right)\right\|_{L^{2}\left(\mathbb{R}_{+}^{3}\right)} .
$$

Similarly, we obtain

$$
\left\|\operatorname{div}_{\Gamma}\left(E^{1} \times e_{3}\right)-\operatorname{div}_{\Gamma}\left(\hat{E}^{1} \times e_{3}\right)\right\|_{H^{-1 / 2}\left(\mathbb{R}_{0}^{3}\right)} \leq C\left\|\left(J_{e}, J_{m}, \hat{J}_{e}, \hat{J}_{m}\right)\right\|_{L^{2}\left(\mathbb{R}_{+}^{3}\right)} .
$$

Using (3.45)-(3.48), without loss of generality, one might assume that $J_{e}=J_{m}=$ $\hat{J}_{e}=\hat{J}_{m}=0$ in $\mathbb{R}_{+}^{3}$.

Combining (3.35) and (3.39) yields

$$
\left(\left|a_{2}(\xi)\right|^{2}+\left|\hat{a}_{2}(\xi)\right|^{2}\right)\left(|\xi|^{2}+|k|^{2}\right)^{-1 / 2} \leq C\left(|h|^{2}+\left|f_{m}^{\mathcal{F}}\right|^{2}+|g(\xi)|^{2}\right)\left(|\xi|^{2}+|k|^{2}\right)^{-1 / 2} .
$$

From the definition of $g$ and $h$, we obtain

$$
\begin{aligned}
& \text { (3.49) } \int_{\mathbb{R}^{2}}\left(\left|a_{2}(\xi)\right|^{2}+\left|\hat{a}_{2}(\xi)\right|^{2}\right)\left(|\xi|^{2}+|k|^{2}\right)^{-1 / 2} d \xi \\
& \leq C\left(\int_{\mathbb{R}^{2}}\left|\left(f_{e}^{\mathcal{F}}, f_{m}^{\mathcal{F}}\right)(\xi)\right|^{2}\left(|\xi|^{2}+|k|^{2}\right)^{-1 / 2} d \xi+\frac{1}{|k|^{2}}\left\|\left(\operatorname{div}_{\Gamma} f_{e}, \operatorname{div}_{\Gamma} f_{m}\right)\right\|_{H^{-1 / 2}\left(\mathbb{R}_{0}^{3}\right)}^{2}\right) .
\end{aligned}
$$

Similarly, we reach

$$
\begin{aligned}
& (3.50) \quad \int_{\mathbb{R}^{2}}\left(\left|a_{1}(\xi)\right|^{2}+\left|\hat{a}_{1}(\xi)\right|^{2}\right)\left(|\xi|^{2}+|k|^{2}\right)^{-1 / 2} d \xi \\
& \leq C\left(\int_{\mathbb{R}^{2}}\left|\left(f_{e}^{\mathcal{F}}, f_{m}^{\mathcal{F}}\right)(\xi)\right|^{2}\left(|\xi|^{2}+|k|^{2}\right)^{-1 / 2} d \xi+\frac{1}{|k|^{2}}\left\|\left(\operatorname{div}_{\Gamma} f_{e}, \operatorname{div}_{\Gamma} f_{m}\right)\right\|_{H^{-1 / 2}\left(\mathbb{R}_{0}^{3}\right)}^{2}\right) .
\end{aligned}
$$

On the other hand, from (3.28), (3.29), (3.31), and (3.32), we have

$$
\int_{\mathbb{R}_{+}^{3}}|k|^{2}|(E, \hat{E})|^{2} d x \leq C \int_{\mathbb{R}^{2}}|k|^{2}|(a(\xi), \hat{a}(\xi))|^{2}\left(|\xi|^{2}+|k|^{2}\right)^{-1 / 2} d \xi .
$$

Combining (3.49), (3.50), and (3.51) yields

$$
\begin{aligned}
& \quad \int_{\mathbb{R}_{+}^{3}}|k|^{2}|(E, \hat{E})|^{2} d x \\
& \leq C\left(|k|^{2} \int_{\mathbb{R}^{2}}\left|\left(f_{e}^{\mathcal{F}}, f_{m}^{\mathcal{F}}\right)(\xi)\right|^{2}\left(|\xi|^{2}+|k|^{2}\right)^{-1 / 2} d \xi+\left\|\left(\operatorname{div}_{\Gamma} f_{e}, \operatorname{div}_{\Gamma} f_{m}\right)\right\|_{H^{-1 / 2}\left(\mathbb{R}_{0}^{3}\right)}^{2}\right) .
\end{aligned}
$$

Similarly, we obtain

$$
\int_{\mathbb{R}_{+}^{3}}|k|^{2}|(H, \hat{H})|^{2} d x
$$

$$
\leq C\left(|k|^{2} \int_{\mathbb{R}^{2}}\left|\left(f_{e}^{\mathcal{F}}, f_{m}^{\mathcal{F}}\right)(\xi)\right|^{2}\left(|\xi|^{2}+|k|^{2}\right)^{-1 / 2} d \xi+\left\|\left(\operatorname{div}_{\Gamma} f_{e}, \operatorname{div}_{\Gamma} f_{m}\right)\right\|_{H^{-1 / 2}\left(\mathbb{R}_{0}^{3}\right)}^{2}\right) .
$$

Assertion (3.17) now follows from (3.52) and (3.53).

The existence and uniqueness of $(E, H, \hat{E}, \hat{H})$ follow from the computations given above. The proof is complete.

Copyright $@$ by SIAM. Unauthorized reproduction of this article is prohibited. 
As a consequence of Proposition 3.1, we obtain the following.

Corollary 3.1. Let $\gamma>0, k \in \mathbb{C}$ with $\left|\Im\left(k^{2}\right)\right| \geq \gamma|k|^{2}$, and $|k| \geq 1$, and let $\varepsilon, \mu, \hat{\varepsilon}, \hat{\mu} \in\left[L^{\infty}\left(\mathbb{R}_{+}^{3}\right)\right]^{3 \times 3}$ be symmetric, uniformly elliptic, and of class $C^{1}$. Let $\Lambda \geq 1$ be such that

$$
\Lambda^{-1} \leq \varepsilon, \mu, \hat{\varepsilon}, \hat{\mu} \leq \Lambda \text { in } B_{1} \cap \mathbb{R}_{+}^{3} \quad \text { and } \quad\|(\varepsilon, \mu, \hat{\varepsilon}, \hat{\mu})\|_{C^{1}\left(\mathbb{R}_{+}^{3} \cap B_{1}\right)} \leq \Lambda .
$$

Assume that $\varepsilon(0), \hat{\varepsilon}(0), \mu(0), \hat{\mu}(0)$ are isotropic, and for some $\Lambda_{1} \geq 0$

$$
|\varepsilon(0)-\hat{\varepsilon}(0)| \geq \Lambda_{1}, \quad|\mu(0)-\hat{\mu}(0)| \geq \Lambda_{1}, \quad \text { and } \quad|\varepsilon(0) / \mu(0)-\hat{\varepsilon}(0) / \hat{\mu}(0)| \geq \Lambda_{1} .
$$

Let $J_{e}, J_{m}, \hat{J}_{e}, \hat{J}_{m} \in L^{2}\left(\mathbb{R}_{+}^{3}\right)$ and assume that $(E, H, \hat{E}, \hat{H}) \in\left[L^{2}\left(\mathbb{R}^{3}\right)\right]^{12}$ is a solution of (3.15) and (3.16) with $f_{e}=f_{m}=0$. There exist $0<r_{0}<1$ and $k_{0}>1$ depending only on $\gamma, \Lambda$, and $\Lambda_{1}$ such that if the supports of $E, H, \hat{E}, \hat{H}$ are in $B_{r_{0}} \cap \overline{\mathbb{R}_{+}^{3}}$ and $|k| \geq k_{0}$, then

$$
|k|\|(E, H, \hat{E}, \hat{H})\|_{L^{2}\left(\mathbb{R}_{+}^{3}\right)} \leq C\left\|\left(J_{e}, J_{m}, \hat{J}_{e}, \hat{J}_{m}\right)\right\|_{L^{2}\left(\mathbb{R}_{+}^{3}\right)} .
$$

We also have, if $J_{e}, J_{m}, \hat{J}_{e}, \hat{J}_{m} \in H\left(\operatorname{div}, \mathbb{R}_{+}^{3}\right)$ and $J_{e, 3}-\hat{J}_{e, 3}, J_{m, 3}-\hat{J}_{m, 3} \in H^{1 / 2}\left(\mathbb{R}_{0}^{3}\right)$,

$$
\begin{aligned}
&\|(E, H, \hat{E}, \hat{H})\|_{H^{1}\left(\mathbb{R}_{+}^{3}\right)}+|k|\|(E, H, \hat{E}, \hat{H})\|_{L^{2}\left(\mathbb{R}_{+}^{3}\right)} \\
& \leq C\left(\left\|\left(J_{e}, J_{m}, \hat{J}_{e}, \hat{J}_{m}\right)\right\|_{L^{2}\left(\mathbb{R}_{+}^{3}\right)}+\frac{1}{|k|}\left\|\left(\operatorname{div} J_{e}, \operatorname{div} J_{m}, \operatorname{div} \hat{J}_{e}, \operatorname{div} \hat{J}_{m}\right)\right\|_{L^{2}\left(\mathbb{R}_{+}^{3}\right)}\right. \\
&\left.+\frac{1}{|k|}\left\|\left(J_{e, 3}-\hat{J}_{e, 3}, J_{m, 3}-\hat{J}_{m, 3}\right)\right\|_{H^{1 / 2}\left(\mathbb{R}_{0}^{3}\right)}\right) .
\end{aligned}
$$

Here $C$ denotes a positive constant depending only on $\gamma, \Lambda$, and $\Lambda_{1}$.

Remark 3.2. The constant $C$ in (3.55) is independent of $k$. Concerning the isotropic properties of $\varepsilon, \mu, \hat{\varepsilon}, \hat{\mu}$, we emphasize here that $\varepsilon, \mu, \hat{\varepsilon}, \hat{\mu}$ are not required to be isotropic in $B_{1} \cap \overline{\mathbb{R}_{+}^{3}}$; we only assume that $\varepsilon(0), \mu(0), \hat{\varepsilon}(0), \hat{\mu}(0)$ are.

Here and in what follows $B_{r}$ for $r>0$ denotes the ball of radius $r$ centered at the origin.

Proof. We only prove (3.55). The proof of (3.54) is in the same spirit and even easier, and omitted. We rewrite (3.15) under the form

$$
\left\{\begin{array} { c } 
{ \nabla \times E = k \mu ( 0 ) H + J _ { e } ^ { 1 } \text { in } \mathbb { R } _ { + } ^ { 3 } , } \\
{ \nabla \times H = - k \varepsilon ( 0 ) E + J _ { m } ^ { 1 } \text { in } \mathbb { R } _ { + } ^ { 3 } , }
\end{array} \quad \left\{\begin{array}{c}
\nabla \times \hat{E}=k \hat{\mu}(0) \hat{H}+\hat{J}_{e}^{1} \text { in } \mathbb{R}_{+}^{3}, \\
\nabla \times \hat{H}=-k \hat{\varepsilon}(0) \hat{E}+\hat{J}_{m}^{1} \text { in } \mathbb{R}_{+}^{3},
\end{array}\right.\right.
$$

where, in $\mathbb{R}_{+}^{3}$,

$$
\begin{array}{ll}
J_{e}^{1}(x)=J_{e}(x)+k(\mu(x)-\mu(0)) H(x), & J_{m}^{1}(x)=J_{m}(x)-k(\varepsilon(x)-\varepsilon(0)) E(x), \\
\hat{J}_{e}^{1}(x)=\hat{J}_{e}(x)+k(\hat{\mu}(x)-\hat{\mu}(0)) \hat{H}(x), & \hat{J}_{m}^{1}(x)=\hat{J}_{m}(x)-k(\hat{\varepsilon}(x)-\hat{\varepsilon}(0)) \hat{E}(x) .
\end{array}
$$

From Proposition 3.1, we obtain

$$
\begin{aligned}
C\left(\|(E, H, \hat{E}, \hat{H})\|_{H^{1}\left(\mathbb{R}_{+}^{3}\right)}+|k|\|(E, H, \hat{E}, \hat{H})\|_{L^{2}\left(\mathbb{R}_{+}^{3}\right)}\right) & \\
\leq\left\|\left(J_{e}^{1}, J_{m}^{1}, \hat{J}_{e}^{1}, \hat{J}_{m}^{1}\right)\right\|_{L^{2}\left(\mathbb{R}_{+}^{3}\right)}+\frac{1}{|k|} \| & \left(\operatorname{div} J_{e}^{1}, \operatorname{div} J_{m}^{1}, \operatorname{div} \hat{J}_{e}^{1}, \operatorname{div} \hat{J}_{m}^{1}\right) \|_{L^{2}\left(\mathbb{R}_{+}^{3}\right)} \\
& +\frac{1}{|k|}\left\|\left(J_{e, 3}^{1}-\hat{J}_{e, 3}^{1}, J_{m, 3}^{1}-\hat{J}_{m, 3}^{1}\right)\right\|_{H^{1 / 2}\left(\mathbb{R}_{0}^{3}\right)} .
\end{aligned}
$$

Copyright (c) by SIAM. Unauthorized reproduction of this article is prohibited. 
On the other hand, from the definition of $\left(J_{e}^{1}, J_{m}^{1}, \hat{J}_{e}^{1}, \hat{J}_{m}^{1}\right)$, one has

$\left\|\left(J_{e}^{1}, J_{m}^{1}, \hat{J}_{e}^{1}, \hat{J}_{m}^{1}\right)\right\|_{L^{2}\left(\mathbb{R}_{+}^{3}\right)} \leq C\left\|\left(J_{e}, J_{m}, \hat{J}_{e}, \hat{J}_{m}\right)\right\|_{L^{2}\left(\mathbb{R}_{+}^{3}\right)}+C r_{0}|k|\|(E, H, \hat{E}, \hat{H})\|_{L^{2}\left(\mathbb{R}_{+}^{3}\right)}$,

$$
\begin{aligned}
& \frac{1}{|k|}\left\|\left(\operatorname{div} J_{e}^{1}, \operatorname{div} J_{m}^{1}, \operatorname{div} \hat{J}_{e}^{1}, \operatorname{div} \hat{J}_{m}^{1}\right)\right\|_{L^{2}\left(\mathbb{R}_{+}^{3}\right)} \\
& \quad \leq \frac{C}{|k|}\left\|\left(\operatorname{div} J_{e}, \operatorname{div} J_{m}, \operatorname{div} \hat{J}_{e}, \operatorname{div} \hat{J}_{m}\right)\right\|_{L^{2}\left(\mathbb{R}_{+}^{3}\right)} \\
& \quad+C\|(E, H, \hat{E}, \hat{H})\|_{L^{2}\left(\mathbb{R}_{+}^{3}\right)}+C r_{0}\|(\nabla E, \nabla H, \nabla \hat{E}, \nabla \hat{H})\|_{L^{2}\left(\mathbb{R}_{+}^{3}\right)},
\end{aligned}
$$

and

$$
\begin{array}{r}
\frac{1}{|k|}\left\|\left(J_{e, 3}^{1}-\hat{J}_{e, 3}^{1}, J_{m, 3}^{1}-\hat{J}_{m, 3}^{1}\right)\right\|_{H^{1 / 2}\left(\mathbb{R}_{0}^{3}\right)} \leq \frac{C}{|k|}\left\|\left(J_{e, 3}-\hat{J}_{e, 3}, J_{m, 3}-\hat{J}_{m, 3}\right)\right\|_{H^{1 / 2}\left(\mathbb{R}_{0}^{3}\right)} \\
+C r_{0}\|(E, H, \hat{E}, \hat{H})\|_{H^{1}\left(\mathbb{R}_{+}^{3}\right)}+C\|(E, H, \hat{E}, \hat{H})\|_{L^{2}\left(\mathbb{R}_{+}^{3}\right)} .
\end{array}
$$

Here in the last inequality, we involved the trace theory and used

$$
\begin{aligned}
\|((\mu(x)-\mu(0)) H,(\varepsilon(x)- & \varepsilon(0)) E,(\hat{\mu}(x)-\hat{\mu}(0)) \hat{H},(\hat{\varepsilon}(x)-\hat{\varepsilon}(0)) \hat{E}) \|_{H^{1}\left(\mathbb{R}_{+}^{3}\right)} \\
& \leq C r_{0}\|(E, H, \hat{E}, \hat{H})\|_{H^{1}\left(\mathbb{R}_{+}^{3}\right)}+C\|(E, H, \hat{E}, \hat{H})\|_{L^{2}\left(\mathbb{R}_{+}^{3}\right)} .
\end{aligned}
$$

Combining (3.56)-(3.59) yields

$$
\begin{aligned}
& C\left(\|(E, H, \hat{E}, \hat{H})\|_{H^{1}\left(\mathbb{R}_{+}^{3}\right)}+|k|\|(E, H, \hat{E}, \hat{H})\|_{L^{2}\left(\mathbb{R}_{+}^{3}\right)}\right) \\
& \leq\left\|\left(J_{e}, J_{m}, \hat{J}_{e}, \hat{J}_{m}\right)\right\|_{L^{2}\left(\mathbb{R}_{+}^{3}\right)}+\frac{1}{|k|}\left\|\left(\operatorname{div} J_{e}, \operatorname{div} J_{m}, \operatorname{div} \hat{J}_{e}, \operatorname{div} \hat{J}_{m}\right)\right\|_{L^{2}\left(\mathbb{R}_{+}^{3}\right)} \\
& \quad+\frac{1}{|k|}\left\|\left(J_{e, 3}-\hat{J}_{e, 3}, J_{m, 3}-\hat{J}_{m, 3}\right)\right\|_{H^{1 / 2}\left(\mathbb{R}_{0}^{3}\right)} \\
& +\left(|k| r_{0}+1\right)\|(E, H, \hat{E}, \hat{H})\|_{L^{2}\left(\mathbb{R}_{+}^{3}\right)}+r_{0}\|(E, H, \hat{E}, \hat{H})\|_{H^{1}\left(\mathbb{R}_{+}^{3}\right)} .
\end{aligned}
$$

Fix $r_{0}=\min \{C / 4,1 / 4\}$, where $C$ is the constant in (3.60). Take $k_{0}$ such that $k_{0} r_{0} \geq 1$. One then can absorb the last two terms of the RHS of (3.60) by the LHS. The conclusion then follows.

4. Proof of Theorem 1.1. In this section, we give the proof of Theorem 1.1. We begin with a result which yields the uniqueness and the stability of (1.7) and (1.8).

Proposition 4.1. Let $\gamma>0$, and let $\varepsilon, \mu, \hat{\varepsilon}, \hat{\mu} \in\left[L^{\infty}(\Omega)\right]^{3 \times 3}$ be symmetric. Assume that there exist $\Lambda \geq 1, \Lambda_{1}>0$, and $s_{0}>0$ such that

$$
\begin{gathered}
\Lambda^{-1} \leq \varepsilon, \mu, \hat{\varepsilon}, \hat{\mu} \leq \Lambda \text { a.e. in } \Omega, \quad\|(\varepsilon, \mu, \hat{\varepsilon}, \hat{\mu})\|_{C^{1}\left(\bar{\Omega}_{s_{0}}\right)} \leq \Lambda, \\
\varepsilon, \mu, \hat{\varepsilon}, \hat{\mu} \text { are isotropic on } \partial \Omega
\end{gathered}
$$

Copyright $@$ by SIAM. Unauthorized reproduction of this article is prohibited. 
and, for $x \in \partial \Omega$,

$$
|\varepsilon(x)-\hat{\varepsilon}(x)| \geq \Lambda_{1}, \quad|\mu(x)-\hat{\mu}(x)| \geq \Lambda_{1}, \quad \text { and } \quad|\varepsilon(x) / \mu(x)-\hat{\varepsilon}(x) / \hat{\mu}(x)| \geq \Lambda_{1} .
$$

There exist two positive constants $k_{0} \geq 1$ and $C>0$ depending only on $\Lambda, \Lambda_{1}$, $s_{0}, \gamma$, and $\Omega$ such that for $k \in \mathbb{C}$ with $\left|\Im\left(k^{2}\right)\right| \geq \gamma|k|^{2}$ and $|k| \geq k_{0}$, for every $\left(J_{e}, J_{m}, \hat{J}_{e}, \hat{J}_{m}\right) \in\left[L^{2}(\Omega)\right]^{12}$ and for every solution $(E, H, \hat{E}, \hat{H}) \in\left[L^{2}(\Omega)\right]^{12}$ of

$$
\begin{aligned}
& \left\{\begin{array} { c } 
{ \nabla \times E = k \mu H + J _ { e } \text { in } \Omega , } \\
{ \nabla \times H = - k \varepsilon E + J _ { m } \text { in } \Omega , }
\end{array} \quad \left\{\begin{array}{c}
\nabla \times \hat{E}=k \hat{\mu} \hat{H}+\hat{J}_{e} \text { in } \Omega, \\
\nabla \times \hat{H}=-k \hat{\varepsilon} \hat{E}+\hat{J}_{m} \text { in } \Omega,
\end{array}\right.\right. \\
& (\hat{E}-E) \times \nu=0 \text { on } \partial \Omega, \quad \text { and } \quad(\hat{H}-H) \times \nu=0 \text { on } \partial \Omega,
\end{aligned}
$$

we have

$$
|k|\|(E, H, \hat{E}, \hat{H})\|_{L^{2}(\Omega)} \leq C\left\|\left(J_{e}, J_{m}, \hat{J}_{e}, \hat{J}_{m}\right)\right\|_{L^{2}(\Omega)} .
$$

Moreover, if $\left(J_{e}, J_{m}, \hat{J}_{e}, \hat{J}_{m}\right) \in[H(\operatorname{div}, \Omega)]^{4}$ with $\left(J_{e} \cdot \nu-\hat{J}_{e} \cdot \nu, J_{m} \cdot \nu-\hat{J}_{m} \cdot \nu\right) \in$ $\left[H^{1 / 2}(\partial \Omega)\right]^{2}$, then

$$
|k|\|(E, H, \hat{E}, \hat{H})\|_{L^{2}(\Omega)}+\|(E, H, \hat{E}, \hat{H})\|_{H^{1}\left(\Omega_{s_{0} / 2}\right)} \leq C\left\|\left(J_{e}, J_{m}, \hat{J}_{e}, \hat{J}_{m}\right)\right\|_{L^{2}(\Omega)}
$$

$+\frac{C}{|k|}\left\|\left(\operatorname{div} J_{e}, \operatorname{div} J_{m}, \operatorname{div} \hat{J}_{e}, \operatorname{div} \hat{J}_{m}\right)\right\|_{L^{2}(\Omega)}+\frac{C}{|k|}\left\|\left(J_{e} \cdot \nu-\hat{J}_{e} \cdot \nu, J_{m} \cdot \nu-\hat{J}_{m} \cdot \nu\right)\right\|_{H^{1 / 2}(\Omega)}$.

Recall that $\Omega_{s}$ is given in (2.1).

Proof. We only prove (4.4). The proof of (4.3) is similar to the one of (4.4) and even easier, and omitted. We use local charts for $\Gamma=\partial \Omega$. In what follows, we denote $Q=(-1,1)^{3}, Q_{+}=Q \cap \mathbb{R}_{+}^{3}$, and $Q_{0}=Q \cap \mathbb{R}_{0}^{3}$.

Let $m \geq 1$ and let $\varphi_{\ell} \in C_{c}^{2}\left(\mathbb{R}^{3}\right), U_{\ell} \subset \mathbb{R}^{3}$ open ball, and $\mathcal{T}_{\ell}: U_{\ell} \rightarrow Q$ with $1 \leq \ell \leq m$ be such that $\mathcal{T}_{\ell}\left(U_{\ell} \cap \Omega\right)=Q_{+}$, and $\mathcal{T}_{\ell}\left(U_{\ell} \cap \Gamma\right)=Q_{0}$, supp $\varphi_{\ell} \Subset U_{\ell}$, and $\Phi=1$ in a neighborhood of $\Gamma$, where

$$
\Phi:=\sum_{\ell=1}^{m} \varphi_{\ell} \text { in } \mathbb{R}^{3}
$$

In what follows, we also assume that the diameter of the support of $\varphi_{\ell}$ is sufficiently small and $\nabla \mathcal{T}_{\ell}\left(\varphi_{\ell}^{-1}(0)\right)$ is a rotation, i.e., $\left(\nabla \mathcal{T}_{\ell} \nabla \mathcal{T}_{\ell}^{T}\right)\left(\varphi_{\ell}^{-1}(0)\right)=I$. Set, in $\Omega \cap U_{\ell}$,

$$
\left(E^{\ell}, H^{\ell}, \hat{E}^{\ell}, \hat{H}^{\ell}\right)=\left(\varphi_{\ell} E, \varphi_{\ell} H, \varphi_{\ell} \hat{E}, \varphi_{\ell} \hat{H}\right)
$$

and

$\left(J_{e}^{\ell}, J_{m}^{\ell}, \hat{J}_{e}^{\ell}, \hat{J}_{m}^{\ell}\right)=\left(\varphi_{\ell} J_{e}+\nabla \varphi_{\ell} \times E, \varphi_{\ell} J_{m}+\nabla \varphi_{\ell} \times H, \varphi_{\ell} \hat{J}_{e}+\nabla \varphi_{\ell} \times \hat{E}, \varphi_{\ell} \hat{J}_{m}+\nabla \varphi_{\ell} \times \hat{H}\right)$.

We have

$$
\left\{\begin{array} { c } 
{ \nabla \times E ^ { \ell } = k \mu H ^ { \ell } + J _ { e } ^ { \ell } \text { in } \Omega \cap U _ { \ell } , } \\
{ \nabla \times H ^ { \ell } = - k \varepsilon E ^ { \ell } + J _ { m } ^ { \ell } \text { in } \Omega \cap U _ { \ell } , }
\end{array} \quad \left\{\begin{array}{r}
\nabla \times \hat{E}^{\ell}=k \hat{\mu} \hat{H}^{\ell}+\hat{J}_{e}^{\ell} \text { in } \Omega \cap U_{\ell}, \\
\nabla \times \hat{H}^{\ell}=-k \hat{\varepsilon} \hat{E}^{\ell}+\hat{J}_{m}^{\ell} \text { in } \Omega \cap U_{\ell},
\end{array}\right.\right.
$$




$$
\left(\hat{E}^{\ell}-E^{\ell}\right) \times \nu=0 \text { on } \partial \Omega \cap U_{\ell}, \quad \text { and } \quad\left(\hat{H}^{\ell}-H^{\ell}\right) \times \nu=0 \text { on } \partial \Omega \cap U_{\ell} .
$$

Given a diffeomorphism $\mathcal{T}$ from an open $D$ onto an open $D^{\prime}$, the following standard notation is used:

$$
\begin{gathered}
\mathcal{T} * u\left(x^{\prime}\right)=\nabla \mathcal{T}(x) u(x), \\
\mathcal{T}_{*} a\left(x^{\prime}\right)=\frac{\nabla \mathcal{T}(x) a(x) \nabla \mathcal{T}^{T}(x)}{\operatorname{det} \nabla \mathcal{T}(x)}, \quad \text { and } \quad \mathcal{T}_{*} j\left(x^{\prime}\right)=\frac{\nabla \mathcal{T}(x) j(x)}{\operatorname{det} \nabla \mathcal{T}(x)}
\end{gathered}
$$

with $x^{\prime}=\mathcal{T}(x)$, for a matrix-valued function $a$, and for vector fields $u$ and $j$ defined in $D$. Set, in $Q_{+}$,

$$
\begin{gathered}
\left(\mathrm{E}^{\ell}, \mathrm{H}^{\ell}, \hat{\mathrm{E}}^{\ell}, \hat{\mathrm{H}}^{\ell}\right)=\left(\mathcal{T}_{\ell} * E^{\ell}, \mathcal{T}_{\ell} * H^{\ell}, \mathcal{T}_{\ell} * \hat{E}^{\ell}, \mathcal{T}_{\ell} * \hat{H}^{\ell}\right), \\
\left(\varepsilon^{\ell}, \mu^{\ell}, \hat{\varepsilon}^{\ell}, \hat{\mu}^{\ell}\right)=\left(\mathcal{T}_{\ell *} \varepsilon, \mathcal{T}_{\ell *} \mu, \mathcal{T}_{\ell *} \hat{\varepsilon}, \mathcal{T}_{\ell *} \hat{\mu}\right), \\
\left(\mathrm{J}_{e}^{\ell}, \mathrm{J}_{m}^{\ell}, \hat{\mathrm{J}}_{e}^{\ell}, \hat{\mathrm{J}}_{m}^{\ell}\right)=\left(\mathcal{T}_{\ell *} J_{e}^{\ell}, \mathcal{T}_{\ell *} J_{m}^{\ell}, \mathcal{T}_{\ell *} \hat{J}_{e}^{\ell}, \mathcal{T}_{\ell *} \hat{J}_{m}^{\ell}\right) .
\end{gathered}
$$

By a change of variables (see, e.g., [23, Lemma 7]),

$$
\begin{gathered}
\left\{\begin{array} { c } 
{ \nabla \times \mathrm { E } ^ { \ell } = k \mu ^ { \ell } \mathrm { H } ^ { \ell } + \mathrm { J } _ { e } ^ { \ell } \text { in } Q _ { + } , } \\
{ \nabla \times \mathrm { H } ^ { \ell } = - k \varepsilon ^ { \ell } \mathrm { E } ^ { \ell } + \mathrm { J } _ { m } ^ { \ell } \text { in } Q _ { + } , }
\end{array} \quad \left\{\begin{array}{c}
\nabla \times \hat{\mathrm{E}}^{\ell}=k \hat{\mu} \hat{\mathrm{H}}^{\ell}+\hat{\mathrm{J}}_{e}^{\ell} \text { in } Q_{+}, \\
\nabla \times \hat{\mathrm{H}}^{\ell}=-k \hat{\varepsilon} \hat{\mathrm{E}}^{\ell}+\hat{\mathrm{J}}_{m}^{\ell} \text { in } Q_{+},
\end{array}\right.\right. \\
\left(\hat{\mathrm{E}}^{\ell}-\mathrm{E}^{\ell}\right) \times \nu=0 \text { on } Q_{0}, \quad \text { and } \quad\left(\hat{\mathrm{H}}^{\ell}-\mathrm{H}^{\ell}\right) \times \nu=0 \text { on } Q_{0} .
\end{gathered}
$$

Since $\nabla \mathcal{T}_{\ell}\left(\varphi_{\ell}^{-1}(0)\right)$ is a rotation, and $\varepsilon, \mu, \hat{\varepsilon}, \hat{\mu}$ are isotropic on $\partial \Omega$, one has

$$
\varepsilon^{\ell}(0), \mu^{\ell}(0), \hat{\varepsilon}^{\ell}(0), \hat{\mu}^{\ell}(0) \text { are isotropic. }
$$

By considering the diameter of $\operatorname{supp} \varphi_{\ell}$ sufficiently small, one can then apply Corollary 3.1 to $\left(\mathrm{E}^{\ell}, \mathrm{H}^{\ell}, \hat{\mathrm{E}}^{\ell}, \hat{\mathrm{H}}^{\ell}\right)$. We then obtain

$$
\begin{aligned}
& C\left(\left\|\left(\mathrm{E}^{\ell}, \mathrm{H}^{\ell}, \hat{\mathrm{E}}^{\ell}, \hat{\mathrm{H}}^{\ell}\right)\right\|_{H^{1}\left(Q_{+}\right)}+|k|\left\|\left(\mathrm{E}^{\ell}, \mathrm{H}^{\ell}, \hat{\mathrm{E}}^{\ell}, \hat{\mathrm{H}}^{\ell}\right)\right\|_{L^{2}\left(Q_{+}\right)}\right) \\
& \leq\left\|\left(\mathrm{J}_{e}^{\ell}, \mathrm{J}_{m}^{\ell}, \hat{\mathrm{J}}_{e}^{\ell}, \hat{\mathrm{J}}_{m}^{\ell}\right)\right\|_{L^{2}\left(Q_{+}\right)}+\frac{1}{|k|}\left\|\left(\operatorname{div} \mathrm{J}_{e}^{\ell}, \operatorname{div} \mathrm{J}_{m}^{\ell}, \operatorname{div} \hat{\mathrm{J}}_{e}^{\ell}, \operatorname{div} \hat{\mathrm{J}}_{m}^{\ell}\right)\right\|_{L^{2}\left(Q_{+}\right)} \\
& +\frac{1}{|k|}\left\|\left(\mathrm{J}_{e}^{\ell} \cdot e_{3}-\hat{\mathrm{J}}_{e}^{\ell} \cdot e_{3}, \mathrm{~J}_{m}^{\ell} \cdot e_{3}-\hat{\mathrm{J}}_{m}^{\ell} \cdot e_{3}\right)\right\|_{H^{1 / 2}\left(Q_{0}\right)} .
\end{aligned}
$$

We have, by [20, Corollary 3.59],

$\left\|\left(\operatorname{div} \mathrm{J}_{e}^{\ell}, \operatorname{div} \mathrm{J}_{m}^{\ell}, \operatorname{div} \hat{\mathrm{J}}_{e}^{\ell}, \operatorname{div} \hat{\mathrm{J}}_{m}^{\ell}\right)\right\|_{L^{2}\left(Q_{+}\right)} \leq C\left\|\left(\operatorname{div} J_{e}^{\ell}, \operatorname{div} J_{m}^{\ell}, \operatorname{div} \hat{J}_{e}^{\ell}, \operatorname{div} \hat{J}_{m}^{\ell}\right)\right\|_{L^{2}\left(\Omega \cap U_{\ell}\right)}$, and we also obtain

$$
\left\|\left(J_{e}^{\ell} \cdot e_{3}-\hat{J}_{e}^{\ell} \cdot e_{3}, J_{m}^{\ell} \cdot e_{3}-\hat{J}_{m}^{\ell} \cdot e_{3}\right)\right\|_{H^{1 / 2}\left(Q_{0}\right)} \leq C \|\left(J_{e}^{\ell} \cdot \nu-\hat{J}_{e}^{\ell} \cdot \nu, J_{m}^{\ell} \cdot \nu-\hat{J}_{m}^{\ell} \cdot \nu \|_{H^{1 / 2}\left(\partial \Omega \cap U_{\ell}\right)} .\right.
$$

We deduce from (4.9) that

$$
\begin{array}{r}
C\left(\left\|\left(E^{\ell}, H^{\ell}, \hat{E}^{\ell}, \hat{H}^{\ell}\right)\right\|_{H^{1}\left(\Omega \cap U_{\ell}\right)}+|k|\left\|\left(E^{\ell}, H^{\ell}, \hat{E}^{\ell}, \hat{H}^{\ell}\right)\right\|_{L^{2}\left(\Omega \cap U_{\ell}\right)}\right) \\
\leq\left\|\left(J_{e}^{\ell}, J_{m}^{\ell}, \hat{J}_{e}^{\ell}, \hat{J}_{m}^{\ell}\right)\right\|_{L^{2}\left(\Omega \cap U_{\ell}\right)}+\frac{1}{|k|}\left\|\left(\operatorname{div} J_{e}^{\ell}, \operatorname{div} J_{m}^{\ell}, \operatorname{div} \hat{J}_{e}^{\ell}, \operatorname{div} \hat{J}_{m}^{\ell}\right)\right\|_{L^{2}\left(\Omega \cap U_{\ell}\right)} \\
+\frac{1}{|k|}\left\|\left(J_{e}^{\ell} \cdot \nu-\hat{J}_{e}^{\ell} \cdot \nu, J_{m} \cdot \nu-\hat{J}_{m} \cdot \nu\right)\right\|_{H^{1 / 2}\left(\partial \Omega \cap U_{\ell}\right)} .
\end{array}
$$

Copyright (c) by SIAM. Unauthorized reproduction of this article is prohibited. 
Take the sum with respect to $\ell$. We then have, for some $\tau_{0}<s_{0} / 4$,

$$
\begin{aligned}
& C\left(\|(E, H, \hat{E}, \hat{H})\|_{H^{1}\left(\Omega_{\tau_{0}}\right)}+|k|\|(E, H, \hat{E}, \hat{H})\|_{L^{2}\left(\Omega_{\tau_{0}}\right)}\right) \\
& \leq\left\|\left(J_{e}, J_{m}, \hat{J}_{e}, \hat{J}_{m}\right)\right\|_{L^{2}(\Omega)}+\frac{1}{|k|}\left\|\left(\operatorname{div} J_{e}, \operatorname{div} J_{m}, \operatorname{div} \hat{J}_{e}, \operatorname{div} \hat{J}_{m}\right)\right\|_{L^{2}(\Omega)} \\
& \quad+\frac{1}{|k|}\left\|\left(J_{e} \cdot \nu-\hat{J}_{e} \cdot \nu, J_{m} \cdot \nu-\hat{J}_{m} \cdot \nu\right)\right\|_{H^{1 / 2}(\partial \Omega)} \\
& \quad+\|(E, H, \hat{E}, \hat{H})\|_{L^{2}\left(\Omega_{s_{0} / 2}\right)}+\frac{1}{|k|}\|(E, H, \hat{E}, \hat{H})\|_{H^{1}\left(\Omega_{s_{0} / 2}\right)} .
\end{aligned}
$$

Applying Lemma 4.1 below, we have

$$
\|(E, H, \hat{E}, \hat{H})\|_{L^{2}\left(\Omega \backslash \Omega_{\tau_{0}}\right)} \leq c_{1} e^{-c_{2}|k|}\|(E, H, \hat{E}, \hat{H})\|_{L^{2}\left(\Omega_{\tau_{0}}\right)}
$$

for some positive constants $c_{1}, c_{2}$ depending only on $\Lambda, \gamma, \tau_{0}$, and $\Omega$. Since $(\varepsilon, \mu, \hat{\varepsilon}, \hat{\mu}) \in$ $C^{1}\left(\Omega_{3 \tau_{0}} \backslash \Omega_{\tau_{0} / 2}\right)$, it follows from (4.1) that

$$
\begin{aligned}
& \|(E, H, \hat{E}, \hat{H})\|_{H^{1}\left(\Omega_{s_{0} / 2} \backslash \Omega_{\tau_{0}}\right)} \leq C|k|\|(E, H, \hat{E}, \hat{H})\|_{L^{2}\left(\Omega_{s_{0}} \backslash \Omega_{\tau_{0} / 2}\right)} \\
& \quad+C\left\|\left(J_{e}, J_{m}, \hat{J}_{e}, \hat{J}_{m}\right)\right\|_{L^{2}(\Omega)}+\frac{C}{|k|}\left\|\left(\operatorname{div} J_{e}, \operatorname{div} J_{m}, \operatorname{div} \hat{J}_{e}, \operatorname{div} \hat{J}_{m}\right)\right\|_{L^{2}(\Omega)} .
\end{aligned}
$$

Taking $k_{0}$ sufficiently large and $|k| \geq k_{0}$, from (4.12) and (4.13), one can absorb the last two terms of the RHS of (4.11) by the LHS of (4.11). We then have

$$
\begin{aligned}
C\left(\|(E, H, \hat{E}, \hat{H})\|_{H^{1}\left(\Omega_{\tau_{0}}\right)}+\right. & \left.|k|\|(E, H, \hat{E}, \hat{H})\|_{L^{2}\left(\Omega_{\tau_{0}}\right)}\right) \\
\leq\left\|\left(J_{e}, J_{m}, \hat{J}_{e}, \hat{J}_{m}\right)\right\|_{L^{2}(\Omega)}+ & \frac{1}{|k|}\left\|\left(\operatorname{div} J_{e}, \operatorname{div} J_{m}, \operatorname{div} \hat{J}_{e}, \operatorname{div} \hat{J}_{m}\right)\right\|_{L^{2}(\Omega)} \\
& +\frac{1}{|k|}\left\|\left(J_{e} \cdot \nu-\hat{J}_{e} \cdot \nu, J_{m} \cdot \nu-\hat{J}_{m} \cdot \nu\right)\right\|_{H^{1 / 2}(\partial \Omega)} .
\end{aligned}
$$

Using (4.12) and (4.14), we derive from (4.13) that

$$
C\|(E, H, \hat{E}, \hat{H})\|_{H^{1}\left(\Omega_{s_{0} / 2} \backslash \Omega_{\tau_{0}}\right)} \leq\left\|\left(J_{e}, J_{m}, \hat{J}_{e}, \hat{J}_{m}\right)\right\|_{L^{2}(\Omega)}
$$

$+\frac{1}{|k|}\left\|\left(\operatorname{div} J_{e}, \operatorname{div} J_{m}, \operatorname{div} \hat{J}_{e}, \operatorname{div} \hat{J}_{m}\right)\right\|_{L^{2}(\Omega)}+\frac{1}{|k|}\left\|\left(J_{e} \cdot \nu-\hat{J}_{e} \cdot \nu, J_{m} \cdot \nu-\hat{J}_{m} \cdot \nu\right)\right\|_{H^{1 / 2}(\partial \Omega)}$.

The conclusion now follows from (4.12), (4.14), and (4.15). The proof is complete.

In the proof of Proposition 4.1, we used the following decay result on the Maxwell equations.

Lemma 4.1. Let $\gamma>0, k \in \mathbb{C}$ with $\left|\Im\left(k^{2}\right)\right| \geq \gamma|k|^{2}$ and $|k| \geq 1$, and let $\varepsilon, \mu \in$ $\left[L^{\infty}(\Omega)\right]^{3 \times 3}$ be symmetric and uniformly elliptic, i.e.,

$$
\Lambda^{-1} \leq \varepsilon, \mu, \hat{\varepsilon}, \hat{\mu} \leq \Lambda
$$

Copyright (c) by SIAM. Unauthorized reproduction of this article is prohibited. 
for some $\Lambda \geq 1$. Given $J_{e}, J_{m} \in L^{2}(\Omega)$, let $(E, H) \in\left[L^{2}(\Omega)\right]^{6}$ be a solution of

$$
\left\{\begin{array}{c}
\nabla \times E=k \mu H+J_{e} \text { in } \Omega, \\
\nabla \times H=-k \varepsilon E+J_{m} \text { in } \Omega .
\end{array}\right.
$$

For all $s>0$, there exists two positive constants $c_{1}$ and $c_{2}$ depending only on $\Lambda, \gamma, s$, and $\Omega$ such that

$$
\|(E, H)\|_{L^{2}\left(\Omega \backslash \Omega_{s}\right)} \leq c_{1} \exp \left(-c_{2}|k|\right)\|(E, H)\|_{L^{2}\left(\Omega_{s}\right)}+c_{1}\left\|\left(J_{e}, J_{m}\right)\right\|_{L^{2}(\Omega)} .
$$

Proof. Let $\left(E^{1}, H^{1}\right) \in\left[L^{2}(\Omega)\right]^{6}$ be the unique solution of

$$
\left\{\begin{array}{c}
\nabla \times E^{1}=k \mu H^{1}+J_{e} \text { in } \Omega, \\
\nabla \times H^{1}=-k \varepsilon E^{1}+J_{m} \text { in } \Omega, \\
E^{1} \times e_{3}=0 \text { on } \partial \Omega .
\end{array}\right.
$$

As in the proof of Lemma 3.1, we have

$$
\left\|\left(E^{1}, H^{1}\right)\right\|_{L^{2}(\Omega)} \leq C\left\|\left(J_{e}, J_{m}\right)\right\|_{L^{2}(\Omega)} .
$$

Considering $\left(E-E^{1}, H-H^{1}\right)$, without loss of generality, one might assume that $J_{e}=J_{m}=0$ in $\Omega$. This is now assumed in what follows.

Fix $\varphi \in C^{2}(\Omega)$ such that $\varphi=c s$ in $\Omega \backslash \Omega_{s}$ and $\varphi=0$ in $\Omega_{s / 2}$, and $|\nabla \varphi| \leq c$ in $\Omega$, where $c$ is a small positive constant defined later. (The smallness of $c$ depends only on $\gamma, \Lambda$, and $\Omega$; it is independent of $s$.) Set $\phi(x)=e^{|k| \varphi(x)}$ and $E^{1}(x)=\phi(x) E(x)$ and $H^{1}(x)=\phi(x) H(x)$ for $x \in \Omega$. We have

$$
\left\{\begin{array}{c}
\nabla \times E^{1}=k \mu H^{1}+J_{e}^{1} \text { in } \Omega, \\
\nabla \times H^{1}=-k \varepsilon E^{1}+J_{m}^{1} \text { in } \Omega,
\end{array}\right.
$$

where

$$
J_{e}^{1}=\nabla \phi \times E \quad \text { and } \quad J_{m}^{1}=\nabla \phi \times H \text { in } \Omega .
$$

Multiplying the first equation with $\bar{H}^{1}$, integrating by parts in $\Omega \backslash \Omega_{\tau}$ for $s / 4<\tau<$ $s / 2$, and using the second equation, we have

$$
\begin{aligned}
\left|\int_{\Omega \backslash \Omega_{\tau}} k\left\langle\mu H^{1}, H^{1}\right\rangle d x+\bar{k} \int_{\Omega \backslash \Omega_{\tau}}\left\langle\varepsilon E^{1}, E^{1}\right\rangle d x\right| \\
\leq \int_{\Omega \backslash \Omega_{\tau}}\left|J_{e}^{1}\right|\left|H^{1}\right|+\left|J_{m}^{1}\right|\left|E^{1}\right| d x+C \int_{\partial\left(\Omega \backslash \Omega_{\tau}\right)}\left(\left|E^{1}\right|^{2}+\left|H^{1}\right|^{2}\right) d x .
\end{aligned}
$$

This yields

$$
\begin{aligned}
& \left.\left|\int_{\Omega \backslash \Omega_{\tau}} k^{2}\left\langle\mu H^{1}, H^{1}\right\rangle d x+\right| k\right|^{2} \int_{\Omega \backslash \Omega_{\tau}}\left\langle\varepsilon E^{1}, E^{1}\right\rangle d x \mid \\
& \quad \leq|k| \int_{\Omega_{\backslash} \Omega_{\tau}}\left|J_{e}^{1}\right|\left|H^{1}\right|+\left|J_{m}^{1}\right|\left|E^{1}\right| d x+C|k| \int_{\partial\left(\Omega \backslash \Omega_{\tau}\right)}\left(\left|E^{1}\right|^{2}+\left|H^{1}\right|^{2}\right) d x .
\end{aligned}
$$

Copyright $@$ ㅇ by SIAM. Unauthorized reproduction of this article is prohibited. 
By the definition of $J_{e}^{1}, J_{m}^{1}$ and of $E^{1}$ and $H^{1}$,

$$
\left|J_{e}^{1}\right| \leq c|k|\left|E^{1}\right|, \quad\left|J_{m}^{1}\right| \leq c|k|\left|H^{1}\right| \text { in } \Omega, \quad \text { and } \quad E^{1}-E=H^{1}-H=0 \text { in } \Omega_{s / 2},
$$

we derive from (4.17) that, for $c$ sufficiently small,

$$
\left.\left|\int_{\Omega \backslash \Omega_{\tau}} k^{2}\left\langle\mu H^{1}, H^{1}\right\rangle d x+\right| k\right|^{2} \int_{\Omega \backslash \Omega_{\tau}}\left\langle\varepsilon E^{1}, E^{1}\right\rangle d x|\leq C| k \mid \int_{\partial\left(\Omega \backslash \Omega_{\tau}\right)}\left(|E|^{2}+|H|^{2}\right) d x .
$$

The conclusion follows by taking $\tau$ such that

$$
\int_{\partial\left(\Omega \backslash \Omega_{\tau}\right)}\left(|E|^{2}+|H|^{2}\right) d x \leq C s^{-1} \int_{\Omega_{s}}\left(|E|^{2}+|H|^{2}\right) d x .
$$

This yields

$$
\int_{\partial\left(\Omega \backslash \Omega_{\tau}\right)}\left(\left|E^{1}\right|^{2}+\left|H^{1}\right|^{2}\right) d x \leq C s^{-1} \int_{\Omega_{s}}\left(|E|^{2}+|H|^{2}\right) d x
$$

and the conclusion follows by the definition of $E^{1}$ and $H^{1}$. The proof is complete.

Remark 4.1. The proof of Lemma 4.1 is quite standard; see, e.g., [15, Theorem 2.2] for a variant dealing with the Helmholtz equation.

We next establish the following.

Proposition 4.2. Assume that the assumptions of Proposition 4.1 hold. There exist two positive constants $k_{0} \geq 1$ and $C>0$ depending only on $\Lambda, \Lambda_{1}, \gamma, s_{0}$, and $\Omega$ such that for $k \in \mathbb{C}$ with $\left|\Im\left(k^{2}\right)\right| \geq \gamma|k|^{2}$ and $|k| \geq k_{0}$, and for every $\left(J_{e}, J_{m}, \hat{J}_{e}, \hat{J}_{m}\right) \in$ $\left[L^{2}(\Omega)\right]^{3}$, there exists a unique solution $(E, H, \hat{E}, \hat{H}) \in\left[L^{2}(\Omega)\right]^{12}$ of (4.1) and (4.2). Moreover,

$$
|k|\|(E, H, \hat{E}, \hat{H})\|_{L^{2}(\Omega)} \leq C\left\|\left(J_{e}, J_{m}, \hat{J}_{e}, \hat{J}_{m}\right)\right\|_{L^{2}(\Omega)} .
$$

Before giving the proof, we denote

$$
\mathbf{H}_{1}(\Omega)=\left\{(u, v) \in[H(\operatorname{curl}, \Omega)]^{2} ;(u-v) \times \nu=0 \text { on } \partial \Omega\right\} .
$$

One can check that $\mathbf{H}_{1}(\Omega)$ is a Hilbert space equipped with the natural scalar product induced from the one of $[H(\operatorname{curl}, \Omega)]^{2}$.

Proof. Applying Proposition 4.1, it suffices to establish the existence. Consider the following equation:

$$
\begin{aligned}
\int_{\Omega}\left\langle\mu^{-1} \nabla\right. & \times E, \nabla \times \varphi\rangle+k^{2}\langle\varepsilon E, \varphi\rangle-\int_{\Omega}\left\langle\hat{\mu}^{-1} \nabla \times \hat{E}, \nabla \times \hat{\varphi}\right\rangle+k^{2}\langle\hat{\varepsilon} \hat{E}, \hat{\varphi}\rangle \\
& =\int_{\Omega}\left\langle\mu^{-1} J_{e}, \nabla \times \varphi\right\rangle+k\left\langle J_{m}, \varphi\right\rangle-\int_{\Omega}\left\langle\hat{\mu}^{-1} \hat{J}_{e}, \nabla \times \hat{\varphi}\right\rangle+k\left\langle\hat{J}_{m}, \hat{\varphi}\right\rangle
\end{aligned}
$$

for all $(\varphi, \hat{\varphi}) \in \mathbf{H}_{1}(\Omega)$.

Copyright $@$ by SIAM. Unauthorized reproduction of this article is prohibited. 
We first note that system (4.1) and (4.2) and system (4.19) are equivalent in the following sense. If $(E, H, \hat{E}, \hat{H}) \in\left[L^{2}(\Omega)\right]^{12}$ is a solution of (4.1) and (4.2), then $(E, \hat{E}) \in \mathbf{H}_{1}(\Omega)$ is a solution of (4.19). Conversely, if $(E, \hat{E}) \in \mathbf{H}_{1}(\Omega)$ is a solution of (4.19), then $(E, H, \hat{E}, \hat{H}) \in\left[L^{2}(\Omega)\right]^{12}$ is a solution of (4.1) and (4.2), where

$$
H=k^{-1} \mu^{-1}\left(\nabla \times E-J_{e}\right) \quad \text { and } \quad \hat{H}=k^{-1} \hat{\mu}^{-1}\left(\nabla \times \hat{E}-\hat{J}_{e}\right) .
$$

The first assertion is clear. Concerning the second assertion, we have, by considering $\varphi, \hat{\varphi} \in C_{c}^{1}(\Omega)$ in $(4.19)$,

$$
\nabla \times\left(\mu^{-1} \nabla \times E\right)+k^{2} \varepsilon E=\nabla \times\left(\mu^{-1} J_{e}\right)+k J_{m} \text { in } \Omega
$$

and

$$
\nabla \times\left(\hat{\mu}^{-1} \nabla \times \hat{E}\right)+k^{2} \hat{\varepsilon} \hat{E}=\nabla \times\left(h \mu^{-1} \hat{J}_{e}\right)+k \hat{J}_{m} \text { in } \Omega .
$$

This yields, by (4.20),

$$
\nabla \times H=-k \varepsilon E^{\delta}+J_{m} \text { in } \Omega \quad \text { and } \quad \nabla \times \hat{H}=-i k \hat{\varepsilon} \hat{E}+\hat{J}_{m} \text { in } \Omega .
$$

This in turn implies, by using (4.19) again, that

$$
(\hat{H}-H) \times \nu=0 \text { on } \partial \Omega .
$$

Therefore, the equivalence is proved.

Consider

$$
a: \mathbf{H}_{1}(\Omega) \times \mathbf{H}_{1}(\Omega) \rightarrow \mathbb{C}
$$

defined as follows. For $(E, \hat{E}) \in \mathbf{H}_{1}(\Omega)$ and $(\varphi, \hat{\varphi}) \in \mathbf{H}_{1}(\Omega), a((E, \hat{E}),(\varphi, \hat{\varphi}))$ is given by the RHS of (4.19). It is clear that $a$ is bilinear and continuous. Define

$$
A: \mathbf{H}_{1}(\Omega) \rightarrow \mathbf{H}_{1}(\Omega)
$$

by, for $(E, \hat{E}) \in \mathbf{H}_{1}(\Omega)$,

$$
\langle A(E, \hat{E}),(\varphi, \hat{\varphi})\rangle_{H(\operatorname{curl}, \Omega)}=a((E, \hat{E}),(\varphi, \hat{\varphi})) \text { for all }(\varphi, \hat{\varphi}) \in \mathbf{H}_{1}(\Omega) .
$$

Applying Proposition 4.1 and using the equivalence of system (4.1) and (4.2) and system (4.19), we have

$$
\|A(E, \hat{E})\|_{H(\operatorname{curl}, \Omega)} \geq C_{k}\|(E, \hat{E})\|_{H(\operatorname{curl}, \Omega)} .
$$

This yields, for all $(E, \hat{E}) \in \mathbf{H}_{1}(\Omega)$,

$$
a((E, \hat{E}), A(E, \hat{E})) \geq C_{k}\|(E, \hat{E})\|_{\mathbf{H}_{1}(\Omega)}\|A(E, \hat{E})\|_{\mathbf{H}_{1}(\Omega)} .
$$

On the other hand, if $a((E, \hat{E}),(\varphi, \hat{\varphi}))=0$ for all $(E, \hat{E}) \in \mathbf{H}_{1}(\Omega)$ and for some $(\varphi, \hat{\varphi}) \in \mathbf{H}_{1}(\Omega)$, then

$$
\varphi=\hat{\varphi}=0 \text { in } \Omega \text {. }
$$

Indeed, this is just a consequence of Proposition 4.1 and the equivalence of system $(4.1)$ and (4.2) and system (4.19) (applied to $\bar{k}$ ). Combining this and (4.21), we obtain the existence of a solution of (4.19) by the Banach-Necas-Babuska theorem. This in 
turn implies the existence of a solution (4.1) and (4.2) using the equivalence of system (4.1) and (4.2) and system (4.19). The proof is complete.

Denote

$$
\begin{gathered}
\mathbf{H}(\Omega)=\left\{(u, v, \hat{u}, \hat{v}) \in\left[L^{2}(\Omega)\right]^{12} ; \operatorname{div}(\varepsilon u)=\operatorname{div}(\mu v)=\operatorname{div}(\hat{\varepsilon} \hat{u})=\operatorname{div}(\hat{\mu} \hat{v})=0 \text { in } \Omega,\right. \\
\text { and } \varepsilon u \cdot \nu-\hat{\varepsilon} \hat{u} \cdot \nu=\mu v \cdot \nu-\hat{\mu} \hat{v} \cdot \nu=0 \text { on } \partial \Omega\},
\end{gathered}
$$

and let

$$
\|(u, v, \hat{u}, \hat{v})\|_{\mathbf{H}(\Omega)}=\|(u, v, \hat{u}, \hat{v})\|_{L^{2}(\Omega)} .
$$

One can check that $\mathbf{H}(\Omega)$ is a Hilbert space with the corresponding scalar product.

We are ready to give the proof of our main theorem.

Proof of Theorem 1.1. Fix $k \in \mathbb{C}$ satisfying the assumptions in Proposition 4.2. Define the operator

$$
\begin{array}{cccc}
T: & \mathbf{H}(\Omega) & \rightarrow & \mathbf{H}(\Omega), \\
\left(J_{e}^{1}, J_{m}^{1}, \hat{J}_{e}^{1}, \hat{J}_{m}^{1}\right) & \mapsto & (E, H, \hat{E}, \hat{H}),
\end{array}
$$

where $(E, H, \hat{E}, \hat{H}) \in\left[L^{2}(\Omega)\right]^{12}$ is the unique solution of (4.1) and (4.2) with

$$
\left(J_{e}, J_{m}, \hat{J}_{e}, \hat{J}_{m}\right)=\left(\mu J_{m}^{1},-\varepsilon J_{e}^{1}, \hat{\mu} \hat{J}_{m}^{1},-\hat{\varepsilon} \hat{J}_{e}^{1}\right) .
$$

Since $\operatorname{div} J_{e}=\operatorname{div} J_{m}=\operatorname{div} \hat{J}_{e}=\operatorname{div} \hat{J}_{m}=0$, it follows that

$$
\operatorname{div}(\varepsilon E)=\operatorname{div}(\mu H)=\operatorname{div}(\hat{\varepsilon} \hat{E})=\operatorname{div}(\hat{\mu} \hat{H})=0 .
$$

We derive that $(E, H, \hat{E}, \hat{H}) \in \mathbf{H}(\Omega)$.

We claim that $T$ is compact. Indeed, this follows from (4.24) and (4.18). By the theory of compact operator (see, e.g., [4]), the spectrum of $T$ is discrete. It is clear that an eigenfunction pair of the ITE problem corresponding to the eigenvalue $\omega$ is an eigenfunction pair of $T$ corresponding to the eigenvalue $k=i \omega$. Hence, the spectrum of the ITE problem is discrete, and the only possible accumulation point of the transmission eigenvalues is $\infty$ since they coincide with the eigenvalues of the inverse of $T$.

Finally, we present the following.

Proof of Proposition 1.1. Proposition 1.1 is just a consequence of Proposition 4.1 by noting that the solution given there is 0 if $\left(J_{e}, J_{m}, \hat{J}_{e}, \hat{J}_{m}\right)=0$.

Acknowledgments. H.-M. Nguyen thanks Fondation des Sciences Mathématiques de Paris (FSMP) for the Chaire d'excellence which allowed him to visit Laboratoire Jacques Louis Lions and Mines ParisTech. This work was completed during this visit.

\section{REFERENCES}

[1] S. Agmon, A. Douglis, And L. Nirenberg, Estimates near the boundary for solutions of elliptic partial differential equations satisfying general boundary conditions. I, Comm. Pure Appl. Math., 12 (1959), pp. 623-727.

[2] S. Agmon, A. Douglis, And L. Nirenberg, Estimates near the boundary for solutions of elliptic partial differential equations satisfying general boundary conditions. II, Comm. Pure Appl. Math., 17 (1964), pp. 35-92. 
[3] A-S. Bonnet-Ben Dhia, L. Chesnel, and H. Haddar, On the use of T-coercivity to study the transmission eigenvalue problem, C. R. Math. Acad. Sci. Paris, 349 (2011), pp. 647-651.

[4] H. Brezis, Functional Analysis, Sobolev Spaces and Partial Differential Equations, Universitext, Springer, New York, 2011.

[5] F. CAkoni, D. Colton, And H. Haddar, Inverse Scattering Theory and Transmission Eigenvalues, CBMS-NSF Regional Conf. Ser. in Appl. Math. 88, SIAM, Philadelphia, 2016.

[6] F. Cakoni, D. Gintides, And H. Haddar, The existence of an infinite discrete set of transmission eigenvalues, SIAM J. Math. Anal., 42 (2010), pp. 237-255.

[7] F. CAKoni, H. Haddar, AND S. Meng, Boundary integral equations for the transmission eigenvalue problem for Maxwell's equations, J. Integral Equations Appl., 27 (2015), pp. 375-406.

[8] F. Cakoni And A. Kirsch, On the transmission eigenvalue problem, Int. J. Comput. Sci. Math., 3 (2010), pp. 142-167.

[9] F. Cakoni, P. Monk, and V. Selgas, Analysis of the linear sampling method for imaging penetrable obstacles in the time domain, Anal. PDE, 2020, to appear.

[10] L. Chesnel, Transmission eigenvalue problem for Maxwell's equations: The T-coercivity as an alternative approach, Inverse Problems, 28 (2012), 065005.

[11] D. Colton And P. Monk, The inverse scattering problem for time-harmonic acoustic waves in an inhomogeneous medium, Quart. J. Mech. Appl. Math., 41 (1988), pp. 97-125.

[12] G. Csato, B. Dacorogna, and S. Sil, On the best constant in Gaffney inequality, J. Funct. Anal., 274 (2018), pp. 461-503.

[13] V. Girault and P-A. Raviart, Finite Element Methods for Navier-Stokes Equations: Theory and Algorithms, Springer Ser. Comput. Math. 5, Springer, New York, 1986.

[14] H. HADDAR, The transmission problem for anisotropic Maxwell's equations and its applications to the inverse problem, Math. Methods Appl. Sci., 27 (2004), pp. 2111-2129.

[15] H. Haddar, P. Joly, and H.-M. NGuyen, Generalized impedance boundary conditions for scattering by strongly absorbing obstacles: The scalar case, Math. Models Methods Appl. Sci., 15 (2005), pp. 1273-1300.

[16] H. HADDAR AND S. MENG, The spectral analysis of the interior transmission eigenvalue problem for Maxwell's equations, J. Math. Pures Appl. (9), 120 (2018), pp. 1-32.

[17] M. Hitrik, K. Krupchyk, P. Ola, and L. PäIVÄrinta, The interior transmission problem and bounds on transmission eigenvalues, Math. Res. Lett., 18 (2011), pp. 279-293.

[18] A. KIRSCH, The denseness of the far field patterns for the transmission problem, IMA J. Appl. Math., 37 (1986), pp. 213-223.

[19] E. LAKSHTANOV AND B. VAINBERG, Ellipticity in the transmission problem in anisotropic media, SIAM J. Math. Anal., 44 (2012), pp. 1165-1174.

[20] P. Monk, Finite Element Methods for Maxwell's Equations, Numer. Math. Sci. Comput., Oxford University Press, New York, 2003.

[21] H.-M. NGUYEn, Asymptotic behavior of solutions to the Helmholtz equations with sign changing coefficients, Trans. Amer. Math. Soc., 367 (2015), pp. 6581-6595.

[22] H.-M. NGUYen, Limiting absorption principle and well-posedness for the Helmholtz equation with sign changing coefficients, J. Math. Pures Appl. (9), 106 (2016), pp. 342-374.

[23] H.-M. NGUYen, Superlensing using complementary media and reflecting complementary media for electromagnetic waves, Adv. Nonlinear Anal., 7 (2018), pp. 449-467.

[24] H.-M. NGUYEN, Cloaking using complementary media for electromagnetic waves, ESAIM Control Optim. Calc. Var., 25 (2019).

[25] H.-M. NGuYen, Cloaking Property of a Plasmonic Structure in Doubly Complementary Media and Three-Sphere Inequalities with Partial Data, 2019, preprint, https://arxiv.org/abs/ 1912.09098 .

[26] H.-M. Nguyen and Q.-H. NGuyen, Discreteness of interior transmission eigenvalues revisited, Calc. Var. Partial Differential Equations, 56 (2017).

[27] H.-M. NGuyen AND S. Sil, Limiting absorption principle and well-posedness for the Maxwell equations with anisotropic sign changing coefficients, Comm. Math. Phys., 379 (2020), pp. 145-176.

[28] L. PÄIvÄrinta And J. Sylvester, Transmission eigenvalues, SIAM J. Math. Anal., 40 (2008), pp. $738-753$.

[29] B. P. Rynne And B. D. Sleeman, The transmission problem and inverse scattering from inhomogeneous media, SIAM J. Math. Anal., 22 (1991) pp. 1755-1762.

[30] L. Robbiano, Spectral analysis of the transmission eigenvalue problem, Inverse Problems, 29 (2013), 104001.

[31] L. Robbiano, Counting function for transmission eigenvalues, Math. Control Relat. Fields, 6 (2016), pp. 167-183. 
[32] J. SYLVESTER, Discreteness of transmission eigenvalues via upper triangular compact operators, SIAM J. Math. Anal., 44 (2012), pp. 341-354.

[33] G. Vodev, Transmission eigenvalue-free regions, Comm. Math. Phys., 336 (2015), pp. 11411166.

[34] G. Vodev, High-frequency approximation of the interior Dirichlet-to-Neumann map and applications to the transmission eigenvalues, Anal. PDE, 11 (2018), pp. 213-236.

Copyright $@$ by SIAM. Unauthorized reproduction of this article is prohibited. 